\title{
SOZIALE REPRÄSENTATIONEN VON PSYCHISCHER KRANKHEIT EIN VERGLEICH DER VORSTELLUNGSSYSTEME VON ÄRZTEN UND LAIEN 1830-1850
}

Obwohl psychische Krankheiten zunehmend durch biologische Ursachen im Körper zu erklären versucht werden, nehmen diese Erkrankungen noch nicht den gleichen Rang wie etwa Infektionen oder Gelenksbrüche ein. Die Äußerungen der Krankheiten rütteln an den Gitterstäben, die die „Normalität“ eingrenzen, und erwecken starke Bilder in uns, die unterschiedlichen Inhaltes sein können und sich vielfach nicht mit dem wissenschaftlichen Verständnis von psychischen Krankheiten vereinbaren lassen.

Diese Untersuchung von Krankenakten - von 30 Frauen und 30 Männern der „Kaiserlich-königlichen Provinzial-Irrenanstalt“ in Hall in Tirol $^{1}$ aus den Jahren 1830 bis 1850 - soll die Wurzeln der unterschiedlichen Vorstellungen von Laien und Experten etwas deutlicher machen. Gerichtsprotokolle, Berichte und Briefe von Erkrankten und ihren Angehörigen wie auch die Erzählungen, die in die Krankengeschichten Eingang fanden, geben dabei Aufschluss über die Sicht der Laien. Medizinische Schlussfolgerungen der Ärzte in der Krankengeschichte, Anamnesen, tägliche und monatliche Berichte auf den Ordinationszetteln in der Anstalt repräsentieren die Vorstellungen der Ärzte. Die wechselseitigen Einflüsse mit einbeziehend soll der Frage nachgegangen werden, ob sich an den verwendeten Formulierungen und Begriffen verschiedene Wahrnehmungsmodelle von psychisch Erkrankten bzw. den Krankheiten ablesen lassen und welche Erklärungsmuster sich für psychische Krankheiten bei Laien und Ärzten finden.

\section{Klärung des Begriffs „Soziale Repräsentationen“}

Die alltäglichen Vorstellungen von psychischer Krankheit werden heute sehr stark durch die Medien geprägt, die in der Vermittlung medizinischer Inhalte mittlerweile eine größere Rolle einnehmen als der mündliche Austausch darüber. Durch die Kommunikation über psychische Krankheiten kommt es zu einer Aufrechterhaltung und Weiterentwicklung von Vorstellungen über dieses Phänomen.

1 Angaben zu dieser Anstalt siehe im Beitrag von Elena TADDEI in diesem Band. 
Gemeinsame Vorstellungen über einen Sachverhalt werden von der Soziologie und Sozialpsychologie als soziale Repräsentationen bezeichnet: Man versteht darunter ein System von Werten, Ideen und Praktiken, die eine zweifache Funktion einnehmen. Zum einen wird damit eine Ordnung geschaffen, die die Individuen befähigt, sich in der materiellen und sozialen Welt zu orientieren und sie zu bewältigen. Zum anderen machen soziale Repräsentationen die Kommunikation zwischen den Mitgliedern einer Gemeinschaft möglich, indem ein Code für sozialen Austausch zur Verfügung gestellt wird. ${ }^{2}$ Es handelt sich also um Gruppenphänomene, die in der täglichen Kommunikation und Aktion gemeinsam konstruiert werden. Dabei geht es nicht um das Bild einer Repräsentation, die sich im Kopf eines einzelnen Individuums, sondern vielmehr zwischen den Köpfen der Individuen befindet - im Sinne eines Gewebes, das von den einzelnen Mitgliedern einer Gemeinschaft stets neu gewoben wird. ${ }^{3}$ Soziale Ereignisse und Gegebenheiten, wie beispielsweise Arbeits- oder Rassenkonflikte, kulturelle Objekte wie Körper, Krankheit und Gesundheit oder psychische Zustände können Inhalt sozialer Repräsentationen sein. ${ }^{4}$

Untersuchungen über den aktuellen Umgang mit psychischer Krankheit haben gezeigt, dass sich die Vorstellungen darüber bei Laien und Experten sehr stark unterscheiden können. Projektive Verfahren, wie etwa die Aufgabe, psychisch kranke Personen zeichnerisch darzustellen, wiesen einen starken Zusammenhang mit übernatürlicher Besessenheit, magisch-heiligen Formen von Geisteskrankheit oder Monstrosität nach, während dieselben Personen in der Befragung überzeugend das medizinische Bild von psychischer Krankheit beschrieben. ${ }^{5}$

Ein ähnliches Auseinanderklaffen von sprachlichen Äußerungen und alltäglichen Praktiken zeigte sich auch in der Untersuchung Denise Jodelets über die alltäglichen Vorstellungen von DorfbewohnerInnen in Frankreich, die aufgrund eines Reformprojektes ehemalige PsychiatriepatientInnen in ihren Privathaushalten beherbergten. Während sich die DorfbewohnerInnen in der Befragung deutlich positiv zu diesem Projekt äußerten, stellte die Beobachtung alltäglicher Umgangsweisen mit den Betroffenen tief liegende Ängste, von psychischer Krankheit angesteckt zu werden, fest: Kleine Kinder wurden zum Beispiel von

2 Serge MOSCOVICI, Foreword. In: Claudine HERZLICH, Health and Illness: A Social Psychological Analysis (London 1973) XI-XIII.

3 Wolfgang WAGNER, Gerard DUVEEN, Robert FARR, Sandra JOVCHELOVITCH, Fabio LORENZI-CIOLDI, Ivana MARKOVÁ, Diana ROSE, Theory and method of social representations. In: Asian Journal of Social Psychology 2 (1999) 95-125, hier 96.

4 Wolfgang WAGNER, Nicky HAYES, Everyday Discourse and Common Sense - The Theory of Social Representations (Basingstoke, Hampshire 2005) 144.

5 Vgl. Annamaria Silvana DE ROSA, The social representations of mental illness in children and adults. In: Willem DOISE, Serge MOSCOVICI (Hg.), Current Issues in European Social Psychology 2 (Cambridge 1987) 47-138. 
den Kranken ferngehalten, separates Geschirr verwendet und die von den Erkrankten benutzte Wäsche getrennt gewaschen. ${ }^{6}$

Da sozialpsychologische Phänomene und Prozesse nur dann gründlich verstanden werden können, wenn sie in die jeweiligen historischen, kulturellen und makrosozialen Rahmenbedingungen eingebunden werden, ist es sinnvoll, solche Phänomene und Prozesse auch in historischer Perspektive zu untersuchen.

\section{Stichprobe}

Kriterien der Auswahl waren zunächst eine Gleichverteilung des Geschlechts und eine Einschränkung des Lebensalters der aufgenommenen PatientInnen auf 20 bis 51 Jahre. Berufstätigkeit begann im landwirtschaftlichen Bereich zwar schon mit durchschnittlich 13 Jahren, aber trotzdem bildet der gewählte Zeitraum mit dem Hauptbereich des Erwachsenenalters die größte Vergleichbarkeit. Wesentlich war eine ungefähr gleichmäßige Verteilung der Akten über die 20 Jahre des Untersuchungszeitraums hinweg und das Vorhandensein der wichtigsten Dokumente (Krankengeschichte und Anamnese). Bevorzugt aufgenommen wurden Krankenakten mit zusätzlichen Dokumenten in Form von Briefen und Berichten der Erkrankten und Angehörigen, Stellungnahmen der Ortsgeistlichen und Gerichtsprotokolle. Die Streuung hinsichtlich der Diagnosen, des Familienstatus (ledig, verheiratet, verwitwet) und der sozialen Herkunft (bäuerlicher Kontext, Handwerk, Handel, Beamtenstand, GutsbesitzerInnen, geistliche Berufe, Ärzte, DienstbotInnen, TagelöhnerInnen, Soldaten, Studierende, Bergbau, Künstler, VagantInnen, Sträflinge) geschah zufällig. Die hohe Ledigenquote bei den DienstbotInnen hing mit der Struktur dieser Arbeit zusammen. Heirateten Mägde oder Knechte, dann schieden sie aus dem Hofverband aus und zogen auf ein eigenes Gut oder in ein Haus ohne Landwirtschaft.

Die Gleichverteilung des Geschlechts in der Stichprobe entspricht nicht der Verteilung der Gesamtaufnahmen des Untersuchungszeitraums, die sich auf 552 Männer und 345 Frauen erstreckte. Das Übergewicht der Männer wurde zeitgenössisch damit erklärt, dass psychisch kranke Frauen viel häufiger in anderen Einrichtungen, wie Schwesternorden oder Spitäler unterkamen. ${ }^{7}$ Erst in der zweiten Hälfte des 19. Jahrhunderts nahm man eine größere Häufigkeit von psychischen Krankheiten bei einem Geschlecht - den Frauen - an. ${ }^{8}$

6 Vgl. Denise JODELET, Madness and social representation (New York 1991).

7 Josef STOLZ, Bemerkungen über die Tirolische Landes-Irren-Anstalt zu Hall (Innsbruck 1869) 25.

8 F. V. ZILLNER, Ueber die größere Häufigkeit von Irrsinnsfällen unter der weiblichen Bevölkerung des Landes Salzburg. In: Mittheilungen der Salzburger Landeskunde (1879) $151-159$. 
Gemessen an der sozialen Zusammensetzung der Tiroler und Vorarlberger Gesellschaft stellte sich die PatientInnenlandschaft der Anstalt differenzierter dar. Die Agrarquote Tirols und Vorarlbergs lag um 1800 bei ca. 90 Prozent und um 1850 noch bei rund 78 Prozent. ${ }^{9}$ Somit entsprechen die Kategorien bäuerlicher Kontext wie auch DienstbotInnen und TagelöhnerInnen, die vor allem in der Landwirtschaft tätig waren, mit einem Gesamtwert von 55,97 Prozent nicht ganz der allgemeinen Bevölkerungsstruktur. Demgegenüber erscheint das Handwerk mit 22,85 Prozent überrepräsentiert. In den Alpenländern erreichte der Anteil an Gewerbe und Industrie erst 1868 einen Wert von 23,30 Prozent. ${ }^{10}$ Zudem wird in dieser Angabe auch der Handel unter Gewerbe begriffen. Das würde bei den Gesamtaufnahmen den Anteil des Handwerks und Handels auf 25,88 Prozent erhöhen. Der Grund für den hohen Wert im Handwerk dürfte daraus resultieren, dass oft gerade dörfliches Handwerk eng mit Landwirtschaft verbunden war, laut Selbstdefinition aber zum Handwerk gezählt wurde. Jedoch lässt sich feststellen, dass passend zur leichten Unterrepräsentativität der Gruppe bäuerlicher Kontext eine im Vergleich zur allgemeinen Tiroler und Vorarlberger Bevölkerung höhere Zahl an Geistlichen, Studenten, Beamten und GutsbesitzerInnen - also Angehörigen der höheren sozialen Schicht - aufgenommen wurde.

Vergleicht man die Stichprobe mit den Gesamtaufnahmen, erscheint die Verteilung im Wesentlichen ähnlich. Abweichungen ergeben sich im bäuerlichen Kontext und im Handwerk durch eine geringere Anzahl an ledigen Männern, während im Handwerk die verheirateten Männer überwiegen. Durch die jeweils geringe Zahl in den einzelnen Kategorien schlugen sich auch die drei Handelsgattinnen auffällig zu Buche. $^{11}$

\section{Wahrnehmung von psychischer Krankheit}

Grundsätzlich lassen sich in der Beschreibung psychischer Krankheiten zwei Modelle unterscheiden. Einmal kam die Krankheit von außen und musste abgewehrt werden. Im anderen Modell entstand die Krankheit im Menschen und brach aus. Jedoch können diese beiden Wahrnehmungsmodelle nicht einfach den Bereichen Laienvorstellungen bzw. Expertenvorstellungen zugeordnet werden. Traditionelle Beschreibungen eines „Einbruchs“ der Krankheit fanden sich auch bei

9 Elisabeth DIETRICH, Die Bevölkerungsentwicklung Tirols im ausgehenden 18. und im 19. Jahrhundert. In: Gesellschaft für Wirtschaftsdokumentationen Ges. m. b. H. (Hg.), Chronik der Tiroler Wirtschaft mit Sonderteil Südtirol (Wien 1992) I 125-139, hier 129.

10 Elisabeth DIETRICH, Wolfgang MEIXNER, Die wirtschaftspolitischen Rahmenbedingungen im ausgehenden 18. und beginnenden 19. Jahrhundert. In: Gesellschaft für Wirtschaftsdokumentationen Ges. m. b. H. (Hg.), Chronik der Tiroler Wirtschaft mit Sonderteil Südtirol (Wien 1992) I 115-124, hier 118.

11 Die für diese Untersuchung ausgewählten Krankenakten des Psychiatrischen Krankenhauses Hall in Tirol (PKH) wurden durch Abkürzung des Familiennamens anonymisiert und mit einer Codierung von $\mathrm{F}$ (weiblich) bzw. M (männlich) 1 bis 30 versehen, die im Folgenden jeweils als Zitatnachweis angeführt wird. 


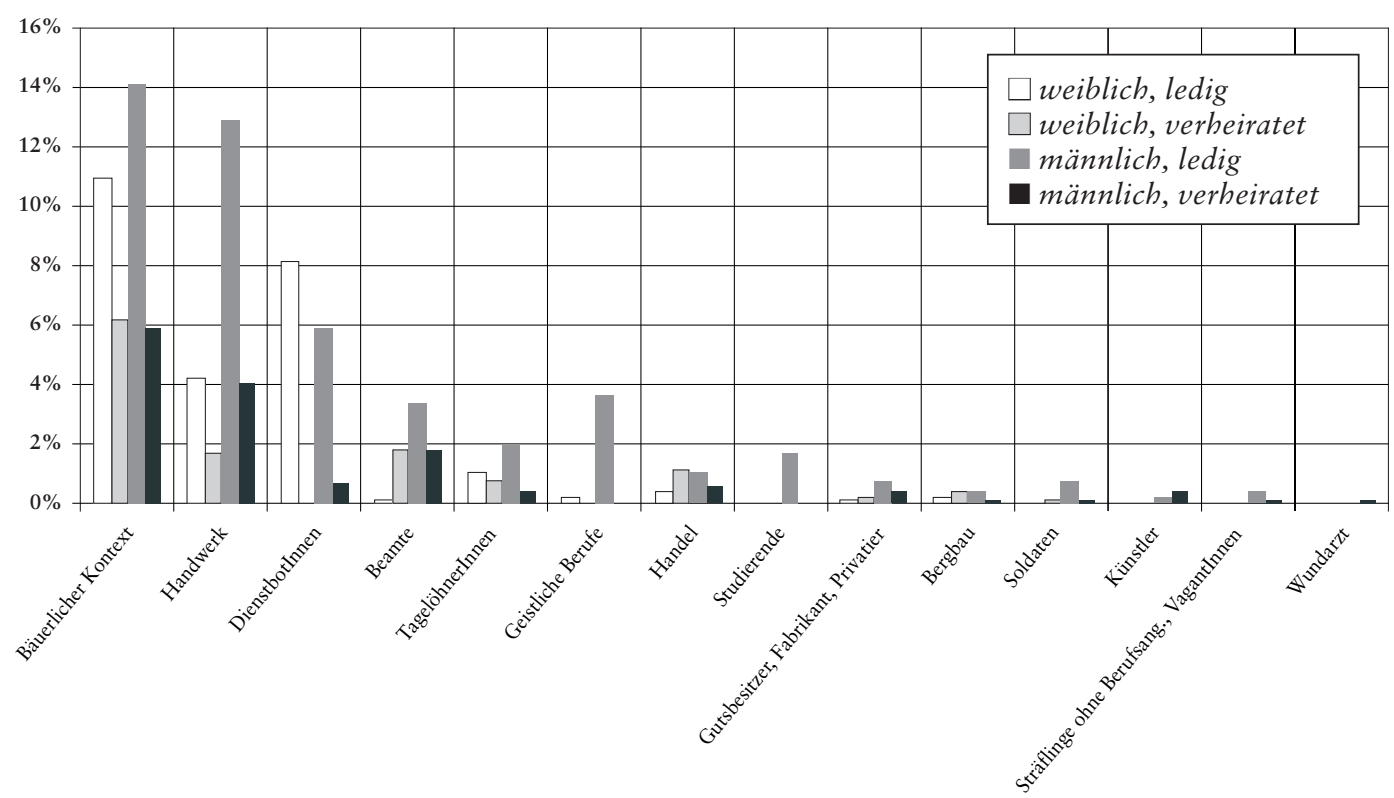

Diagramm 1: Soziale Herkunft der von 1830 bis 1850 aufgenommenen PatientInnen in Prozent. Angaben aus TSCHALLENER, Beschreibung nach S. 119, Tab. Nr. 4 für 1830-1837 bzw. PKH, Aufnabmebuch I für 1838-1850.

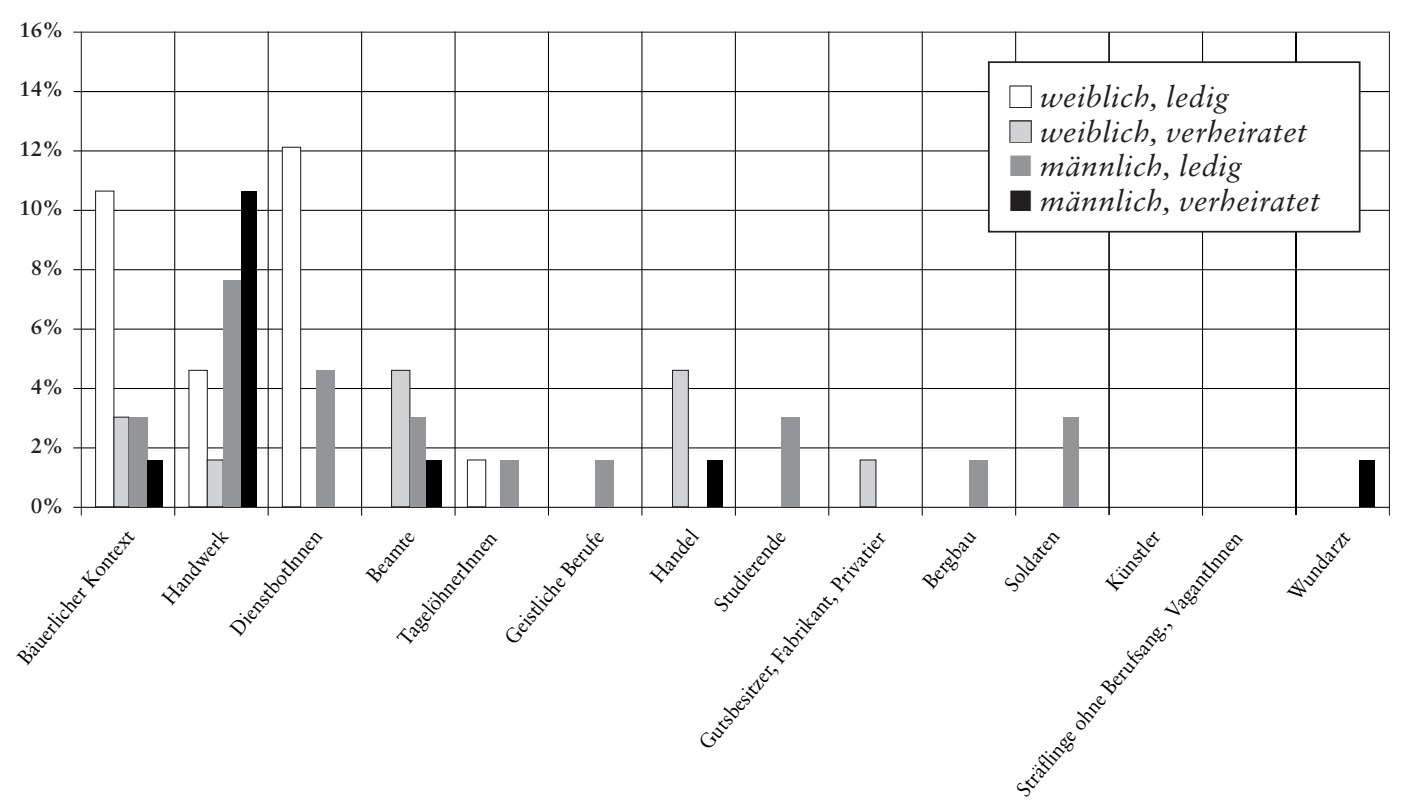

Diagramm 2: Soziale Herkunft der Frauen und Männer der Stichprobe. Da im Aufnahmebuch I auch die wiederholten Aufnahmen extra ausgewiesen und deshalb mitgezählt wurden, ergab sich aufgrund sechs wiederholter Aufnahmen von Frauen und Männern der Stichprobe eine Gesamtzabl von 66. 
den Ärzten. Die Verwendung von Ausdrücken, die auf beide Modelle deuten, durch ein und dieselbe Person zeigen, dass auch schon in dieser Zeit eine Reihe von Ausdrücken feststehend war und in der Verwendung nicht hinterfragt wurde. Trotzdem lassen sich Tendenzen erkennen und bestehende Vorstellungsbilder nachvollziehen. Insgesamt aber scheint sich die Vorstellung eines „Ausbruchs“ - vor allem in der ärztlichen Begrifflichkeit - durchgesetzt zu haben.

Die Ärzte in der Anstalt verwendeten sowohl bei den Frauen (Tab. 1.1) als auch den Männern (Tab. 1.2) deutlich häufiger Begriffe des Ausbruch- als des Einbruchmodells - 24 bzw. 27 Formulierungen gegenüber sieben bzw. acht. Bei den Ärzten, die die Krankengeschichte aufnahmen, hielten sich die verwendeten Begriffe ungefähr die Waage. Sie übernahmen häufig die Ausdrucksweisen der Laien und gingen in den eigenen Interpretationen zum anderen Ausdrucksmodell über. 18 bzw. 22 Aussagen, die auf einen „Ausbruch“ deuten, stehen hier 15 bzw. 18 Formulierungen im Zusammenhang mit „Einbruch“ der Krankheit gegenüber. Betrachtet man schließlich die Laiendokumente, so wurden bei sieben vorhandenen Aussagen von Laien bei den Frauen sechs Formulierungen verwendet, die auf das Vorstellungsmodell des „Einbruchs“ deuten und nur eine, die auf das andere Modell rückschließen lässt.

\section{Austragungsort Mensch: \\ Psychische Krankheit als „Einbruch“ oder „Ausbruch“}

Dabei drehte es sich zunächst um Irrsinn und Wahnsinn selbst. Sie konnten den Menschen befallen oder heimsuchen und dann am Individuum haften. Die Beigabe eines Artikels und die Zuschreibung eines eigenen Charakters verstärkten das Bild eines eigenständigen Wesens, das fähig war, jemanden anzufallen, heimzusuchen und wieder zu verlassen. Sofia S. wurde beispielsweise „[...] Anfangs November l. J. von Wahnsinn befallen, und leidet noch gegenwärtig an dieser Geistes Krankheit." ${ }^{12}$ Die Mutter von Johanna R. war „[...] ohne eine vorausgegangene ihr bekannte Ursache oder Veranlaßung hiezu, von einem Irrsinne befallen..." worden, „[...] deßen Charakter sich in religiöser Schwärmerei, Kleinmuth und Furcht vor Verdammniß aussprach, der aber stets stiller, rubiger Art war. " ${ }^{13}$

$\mathrm{Zu}$ diesem substantivierten Bild der Krankheiten passt es auch, dass man in sie verfallen oder hineinstürzen konnte. Barbara G. sei seit einigen Wochen in einen „[...] mehr weniger anhaltenden, bald stillen, bald aber gewaltsam auftrettenden Wahnsinn verfallen [...] "14 und Peter B. wähnte sich vollends von Gott verlassen, wodurch sein

12 F9, Ärztliches Zeugnis 1835.

13 F11, Krankengeschichte 1835.

14 F5, Krankengeschichte 1830. 
Gemüt laut Krankengeschichte in eine so tiefe Melancholie versetzt wurde, dass es „[...] nur eines geeigneten Zufalls bedurfte, um ibn in tobenden Wabnsinn zu stürzen. " 15 Daraufhin konnte die Geisteskrankheit auch ein Eigenleben entwickeln und den Menschen herumtreiben. Der Dekanprovisor schrieb über die Schwester von Ursula T., dass sie „[...] von religiöser Melancholie getrieben [...]" gewesen sei. ${ }^{16}$ Menschen wurden meist dann als irrsinnig oder wahnsinnig bezeichnet, wenn sie länger an der Krankheit litten, sich ihr Zustand als überdauernder darstellte, also als eine zum Menschen gehörige Eigenschaft begriffen wurde, oder die Betonung nicht auf dem Übergang in die Krankheit lag, sondern auf dem sich zeigenden Zustand. Maria R. sei „[...] nun schon über Sieben Jahre vollkommen Wahnsinnig [... "17 und Franz F. „[...] wirklich wahnsinnig [...]", wobei dieser Wahnsinn „[...] nach Aussage 12 Jahr dauerte. " 18

Wahnsinn, Irrsinn und Geistesverwirrung zeigten sich oft zuerst in Spuren und Anzeichen. Es herrschte ein Kommen und Gehen: Die Spuren kamen, traten in Erscheinung, hafteten am Menschen, konnten vielleicht zum Weichen gebracht werden oder von selbst weggehen. Blieben sie, wurden sie als „fixe Ideen“ bezeichnet, die den Menschen auch beherrschen konnten. Die „[...] irrigen Ansichten [...]“ Joseph G.'s, „[...] daß (er) der Antichrist sey - und so fort, hafteten noch hartnäckig an ihm. "19 Schon bei Elisabeth W.'s erster Entbindung will man

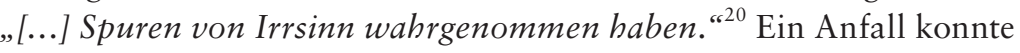
sich auch durch Vorboten ankündigen: „Der Anfall des Wahnsinns hat sich Einigemahl durch Vorbothen, oder Vorzeichen angekündet: denn an jenen Vorabenden, wenn er folgenden Tags ein Attentat zu verüben Willens war, arbeitete er nichts, und aß abends wenig. " 21

Legte man die Krankheit in der Vorstellung stärker in den Menschen hinein, mussten Vernunft und Verstandeskräfte weichen, wenn sich Irrsinn oder fixe Ideen breit gemacht hatten. In der Folge stellte sich die Frage, ob diese wieder zurückkommen würden. So zweifelte der Arzt beispielsweise „[...] an der Rückkehr der Verstandeskräfte [...] “22 Peter B.'s.

Dieses Kommen und Gehen von Spuren, Anzeichen, Vorboten, fixen Ideen, lichten Zwischenräumen und Verstandeskräften war für beide Vorstellungen verwendbar, da sie aus dem Inneren an die Oberfläche

15 M1, Krankengeschichte 1830.

16 F16, Krankengeschichte 1840, wörtlich zitiert vom Bericht des Dekanprovisors.

17 F2, Krankengeschichte 1830.

18 M4, Ärztliches Zeugnis 1830.

19 M6, Krankengeschichte 1831.

20 F8, Krankenberichtigung 1834.

21 M1, Krankengeschichte 1830.

22 M1, Krankengeschichte 1830. 


\begin{tabular}{|c|c|c|c|c|c|c|c|c|c|c|c|c|c|c|c|}
\hline & $\begin{array}{c}\text { Soziale } \\
\text { Herkunft }\end{array}$ & $\begin{array}{l}\text { Bes } \\
\text { der } \\
\text { als, }\end{array}$ & $\begin{array}{l}\text { chreib } \\
\text { Krank } \\
\text { Einbr }\end{array}$ & $\begin{array}{l}\text { ung } \\
\text { heit } \\
\text { uch“ }\end{array}$ & $\begin{array}{l}\text { Bes } \\
\text { der } \\
\text { als, }\end{array}$ & $\begin{array}{l}\text { chreib } \\
\text { Kranl } \\
\text { Ausbr }\end{array}$ & $\begin{array}{l}\text { ung } \\
\text { heit } \\
\text { uch“ }\end{array}$ & $\begin{array}{l}\text { Hinweis } \\
\text { auf } \\
\text { Besessen- }\end{array}$ & $\begin{array}{r}\text { A } \\
\text { Bescl }\end{array}$ & $\begin{array}{l}\text { bfällic } \\
\text { areibu }\end{array}$ & ingen & $\begin{array}{r}\text { Zus } \\
\text { vo } \\
\text { Bo } \\
\text { ur }\end{array}$ & $\begin{array}{l}\text { chreib } \\
\text { Tüc } \\
\text { haftig } \\
\text { d Rac }\end{array}$ & $\begin{array}{l}\text { ung } \\
\text { ke, } \\
\text { keit } \\
\text { he }\end{array}$ & $\begin{array}{l}\text { Zuord- } \\
\text { nung zu } \\
\text { Entste- }\end{array}$ \\
\hline & & LD & KG & $\begin{array}{l}\mathrm{A} / \\
\mathrm{OZ}\end{array}$ & LD & KG & $\begin{array}{l}\mathrm{A} / \\
\mathrm{OZ}\end{array}$ & & LD & KG & \begin{tabular}{|c|}
$\mathrm{A} /$ \\
$\mathrm{OZ}$
\end{tabular} & LD & KG & $\begin{array}{l}\mathrm{A} / \\
\mathrm{OZ}\end{array}$ & $\begin{array}{l}\text { hungska- } \\
\text { tegorien }^{1}\end{array}$ \\
\hline F1 & $\begin{array}{c}\text { Gattin eines } \\
\text { Handelsmanns }\end{array}$ & & $\mathbf{x}$ & & & $\mathbf{x}$ & $\mathbf{x}$ & & & & & & & & 3 \\
\hline F2 & $\begin{array}{l}\text { Schmieds-toch- } \\
\text { ter }\end{array}$ & & & & & & $\mathbf{x}$ & & & & & & & & 2 \\
\hline F3 & $\begin{array}{c}\text { Schichtmeisters- } \\
\text { tochter }\end{array}$ & & $\mathbf{x}$ & $\mathbf{x}$ & & $\mathbf{x}$ & $\mathbf{x}$ & & & & & & $\mathbf{x}$ & & 3 \\
\hline F4 & $\begin{array}{l}\text { Gattin eines } \\
\text { Polizei-Kom- } \\
\text { missars }\end{array}$ & $\mathbf{x}$ & $\mathbf{x}$ & & & & $\mathbf{x}$ & & & & & & & & 2 \\
\hline F5 & Magd & & $\mathbf{x}$ & & & $\mathbf{x}$ & & & & & & $\mathbf{x}$ & $\mathbf{x}$ & $\mathbf{x}$ & 1 \\
\hline F6 & Magd & & $\mathbf{x}$ & $\mathbf{x}$ & & $\mathbf{x}$ & $\mathbf{x}$ & $\mathbf{x}$ & & & $\mathbf{x}$ & & & $\mathbf{x}$ & 3 \\
\hline F7 & Magd & $\mathbf{x}$ & & & & & $\mathbf{x}$ & & & & & $\mathbf{x}$ & & $\mathbf{x}$ & 3 \\
\hline F8 & Bäuerin & $\mathbf{x}$ & $\mathbf{x}$ & & & $\mathbf{x}$ & $\mathbf{x}$ & & & & & & $\mathbf{x}$ & & 3 \\
\hline F9 & $\begin{array}{l}\text { Gattin eines } \\
\text { Handelsmanns }\end{array}$ & & & & & $\mathbf{x}$ & $\mathbf{x}$ & & & & & & & & 3 \\
\hline F10 & $\begin{array}{c}\text { Bettelei/ Dienst- } \\
\text { magd }\end{array}$ & & & & & $\mathbf{x}$ & $\mathbf{x}$ & & & & & & & $\mathbf{x}$ & 1 \\
\hline F11 & Bauerntochter & & $\mathbf{x}$ & & & & $\mathbf{x}$ & $\mathbf{x}$ & & & $\mathbf{x}$ & & & & 2 \\
\hline F12 & Tagelöhnerin & & & & & $\mathbf{x}$ & $\mathbf{x}$ & $\mathbf{x}$ & & & & & & & 3 \\
\hline F13 & $\begin{array}{c}\text { Gattin eines } \\
\text { Kanzlisten }\end{array}$ & & $\mathbf{x}$ & & & $\mathbf{x}$ & $\mathbf{x}$ & $\mathbf{x}$ & & & & & & & 3 \\
\hline F14 & Bäuerin & & $\mathbf{x}$ & $\mathbf{x}$ & & $\mathbf{x}$ & $\mathbf{x}$ & $\mathbf{x}$ & & & & & & & 3 \\
\hline F15 & Bauerntochter & & $\mathbf{x}$ & & & $\mathbf{x}$ & $\mathbf{x}$ & $\mathbf{x}$ & & & & & & & 3 \\
\hline F16 & Bauerntochter & $\mathbf{x}$ & & & & $\mathbf{x}$ & $\mathbf{x}$ & $\mathbf{x}$ & & & & $\mathbf{x}$ & $\mathbf{x}$ & & 1 \\
\hline F17 & Bauerntochter & & & & & $\mathbf{x}$ & $\mathbf{x}$ & & & & & & & & 3 \\
\hline F18 & Tagelöhnerin & $\mathbf{x}$ & $\mathbf{x}$ & & & & & & & & & & & & 1 \\
\hline F19 & Magd & & & & & $\mathbf{x}$ & $\mathbf{x}$ & & & & & & & & 2 \\
\hline F20 & $\begin{array}{c}\text { Gattin eines } \\
\text { Sattlermeisters }\end{array}$ & & & & & & $\mathbf{x}$ & & & & & & & & 3 \\
\hline F21 & Güterbesitzerin & & & & & $\mathbf{x}$ & & $\mathbf{x}$ & & & & & & & 3 \\
\hline F22 & Bauerntochter & & & & & $\mathbf{x}$ & & $\mathbf{x}$ & & & & & & & 2 \\
\hline F23 & Magd & & & & & & $\mathbf{x}$ & & & & & & $\mathbf{x}$ & & 1 \\
\hline F24 & $\begin{array}{l}\text { Witwe eines } \\
\text { Landrichters }\end{array}$ & & & $\mathbf{x}$ & & & $\mathbf{x}$ & & & & & & & & 1 \\
\hline F25 & Magd & & $\mathbf{x}$ & $\mathbf{x}$ & & & $\mathbf{x}$ & & & & & & & & 2 \\
\hline F26 & Wirtschafterin & & $\mathbf{x}$ & & & & $\mathbf{x}$ & $\mathbf{x}$ & & & & & & & 3 \\
\hline F27 & Bauerntochter & & & & & & & & & & & & & & 1 \\
\hline F28 & Bauerntochter & $\mathbf{x}$ & & & $\mathbf{x}$ & & & & & & & & & & 2 \\
\hline F29 & $\begin{array}{l}\text { Handlungs- } \\
\text { besitzerin }\end{array}$ & & $\mathbf{x}$ & $\mathbf{x}$ & & $\mathbf{x}$ & $\mathbf{x}$ & & & & & & & & 3 \\
\hline F30 & Kammerjungfer & & $\mathbf{x}$ & $\mathbf{x}$ & & $\mathbf{x}$ & $\mathbf{x}$ & & & & & & & & 3 \\
\hline & Summen & 6 & 15 & 7 & 1 & 18 & 24 & 10 & 0 & 0 & 2 & 3 & 5 & 4 & \\
\hline
\end{tabular}

Tab. 1.1. Wahrnehmungs- und Beschreibungskategorien von psychischer Krankheit bei Frauen (Krankenakten mit Laiendokumenten grau unterlegt) 
1) Die Einteilung in die Entstehungskategorien (Erklärungsmuster für psychische Krankheiten) - Zuschreibung eines ausschweifenden Lebenswandels, beschriebener Hang zur

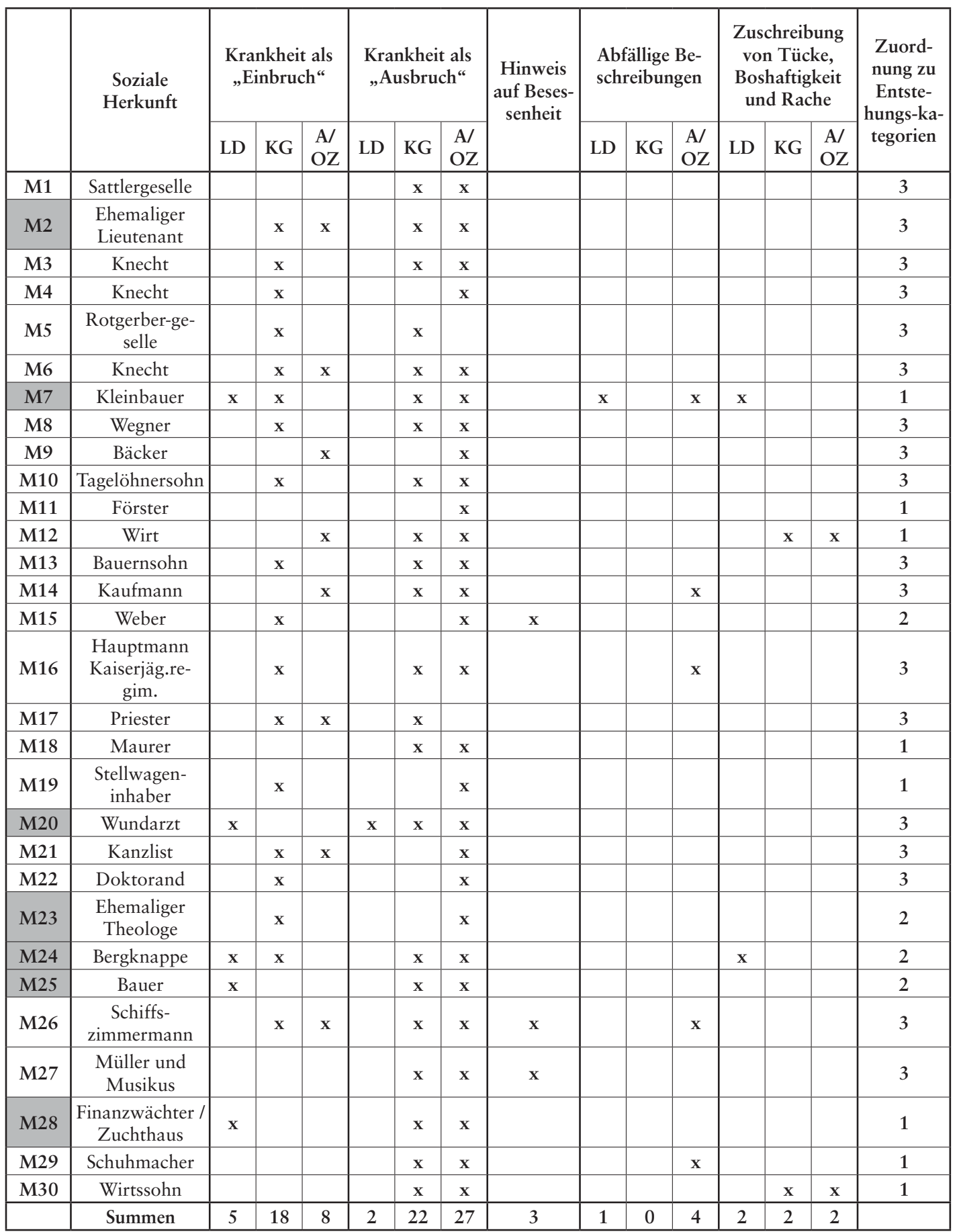

Tab. 1.2. Wahrnehmungs- und Beschreibungskategorien von psychischer Krankheit bei Männern (Krankenakten mit Laiendokumenten grau unterlegt) 
oder von außen her zum Menschen kommen konnten. Die Spuren an den Tag zu legen, wies ebenso wie sich äußernde, zum Vorschein gekommene oder hervortretende Anzeichen deutlicher auf einen Ursprung der Krankheit im Menschen. Die wörtliche Bezeichnung eines „Ausbruchs" von psychischer Krankheit hingegen wurde fast ausschließlich von Ärzten verwendet und kam insgesamt seltener vor. Anna Pr. „[...] zeigte allmählig auffallende Spuren von Geistestrübung unter der Form von Irrsinn [...]" und „[...] verfiel schnell in Störung des Willens als Tobsucht [...]“ bis sie „[...] um die Mitte September l. J. den neuerlichen Ausbruch psychischer Störung auffallend kund gab. “23

Vollends im Inneren des Menschen ist man angelangt, wenn einzelne Vorgänge beschrieben wurden. Die unterschiedlichen Arten von Geisteskrankheiten hielten einander in Schach, übersprangen ihre Schranken und veränderten sich. So hatte Rosina L. „[...] eine Hysterie (entwickelt), welche man selten zu Gesicht bekömmt, und die sogar im Stande war, die Geistesstörung in Bezug auf ihre Äusserung im Schache zu halten, obne daß man behaupten kann, die Figur stehe auch gar nicht mehr auf dem Brette. “24 In der Adventszeit 1829 „[...] übersprang die Melancholie [...] "Peter B.’s plötzlich ihre Schranken, und gieng in tobenden Wahnsinn über. " 25

Plötzlich erschien auch das Ich als Akteur: Durch die nachteilige Wirkung des beängstigten Gemütszustandes auf alle Körperverrichtungen und besonders auf ihr Nervensystem wurde Johanna R.'s psychisches Leben laut der Anamnese in Hall „[...] gebundener, auch sich selber zurückgezogener, der reelen geistigen Welt fremder, ihr Ich gieng in den dunklen Vorstellungen immer mehr unter. “26

Dort im Inneren des Menschen konnte die Krankheit schlummern und wieder erwachen oder nur darauf warten, ins Leben gerufen zu werden. Auch dies war eine typisch ärztliche Ausdrucksweise. Die religiöse Melancholie, die sich durch den Wahn, verdammt zu sein, aussprach, wurde bei Kreszenz S. durch „[...] die Zunahme des frühern geistigen Leidens allmählig verdrängt $u$. endlich ganz eingeschläfert. " ${ }^{27}$ Es „,...] bedürfe nur eines Reizes, um eine solche Krankheit ins Leben zu rufen. ${ }^{28}$

Die ärztlichen Ausdrucksweisen, die sich also zwar nach wie vor der in der Bevölkerung gängigen Begriffe wie „Anfall“, „befallen“ und „haften“ bedienten, deuteten durch neuartige Formulierungen im Zusam-

23 F14, Krankengeschichte 1838.

24 F13, Anamnese 1844.

25 M1, Krankengeschichte 1830.

26 F11, Anamnese 1836.

27 F15, Anamnese 1843.

28 F19, Anamnese 1842. 
menhang mit Geisteskrankheiten wie „Ausbruch“ und Beschreibungen, die sich mit Geschehnissen innerhalb des Menschen beschäftigten, auf das zunehmende Verständnis von psychischen Krankheiten, die in einer inneren Disposition angelegt waren und durch verschiedenste Einflüsse zum „Ausbruch“ kommen konnten.

\section{Wahrnehmung psychisch Kranker als Besessene}

Die Vorstellung der Laien, dass eine Krankheit dem Menschen von außen zustößt, ist dem alten Verständnis von Dämonen, die den Menschen besetzen können, noch sehr nahe. ${ }^{29}$ Mehr oder weniger starke Reflexe davon zeigten sich im Umgang der Laien mit psychischer Krankheit, indem sie verschiedentlich Geistliche um Benediktionen und Exorzismen baten.

Liest man die Krankenakten oberflächlich durch, fallen zunächst kaum Hinweise auf eine Zuschreibung von Besessenheit ins Auge. Ob Angehörige oder Gemeindevorsteher - wer immer erreichen wollte, dass Erkrankte in die Irrenanstalt aufgenommen wurden, betonte die Zuversicht, dass eine Heilung am besten in der Anstalt erfolgen könne. Wenig ratsam schien es, hervorzuheben, dass man eigentlich glaubte, die Heilung vor allem durch Gebet, Beichten und Exorzieren erreichen zu können. Um 1830 stand der Großteil der Ärzte solchen religiösen Deutungen sehr skeptisch gegenüber. So können die weitgehend fehlenden ausdrücklichen Erwähnungen von Besessenheit auch darauf zurückgeführt werden, dass man die Ärzte, die die Krankengeschichte verfassten, günstig stimmen wollte, damit sie eine Aufnahme der Erkrankten in die Anstalt befürworteten. Die Angehörigen konnten jedoch nicht umhin, bei der Frage nach der bisherigen Behandlung zu erwähnen, wenn die oder der Kranke zunächst zu einem Priester geschickt worden war.

In der Stichprobe kamen Hinweise auf mögliche Wahrnehmung psychisch Kranker als Besessene bei zehn Frauen (Tab. 1.1.), jedoch nur bei 3 Männern vor (Tab. 1.2.). Diese Befunde korrespondieren mit der Behandlungshäufigkeit des berühmt gewordenen Exorzisten Johann Joseph Gaßner im bayerischen Raum in der zweiten Hälfte des 18. Jahrhunderts, zu dem ebenfalls vor allem Frauen kamen. Die zeitgenössische Interpretation ging davon aus, dass Frauen wegen einer ihnen zugeschriebenen schwächeren Denkfähigkeit und lebhafteren Empfindsamkeit für diese Prozedur besser empfänglich seien. ${ }^{30}$ Diese ersten Spuren des Gelehrtenmodells der Geschlechtscharaktere ${ }^{31}$, das

29 Heinz SCHOTT, Rainer TÖLLE, Geschichte der Psychiatrie. Krankheitslehren, Irrwege, Behandlungsformen (München 2006) 20-22.

30 Robert JÜTTE, Geschichte der Alternativen Medizin. Von der Volksmedizin zu den unkonventionellen Therapien von heute (München 1996) 88-90.

31 Vgl. Claudia HONEGGER, Die Ordnung der Geschlechter. Die Wissenschaften vom Menschen und das Weib, 1750-1850 (Frankfurt a. M. 1991). 
sich erst auszubilden und verbreiten begann, dürften in dieser Zeit jedoch noch nicht in die einfache Bevölkerung vorgedrungen sein, geschweige denn, dass sich Frauen und Männer auch danach verhielten.

Hinter den „rationalen“ Erklärungen, die Ärzte für die Krankheiten suchten, kam ein Verständnis der Bevölkerung im Umgang mit psychischer Krankheit zum Vorschein, das die Erkrankten vielfach als besessen betrachtete. Man versuchte, diesen Zustand durch Segnungen oder Teufelsaustreibungen durch Geistliche $\mathrm{zu}$ beseitigen. Besprechungen mittels überlieferten religiös gefärbten Sprüchen und Segnungen waren auch bei anderen Erkrankungen üblich und deuten auch dort auf eine Substantivierung der Krankheiten, die kamen und gingen.

Über Ursula T. schrieb der Arzt beispielsweise, dass sie „[...] voll von Aber-besonders Hexenglauben, wie dieser unter dem Volke hierum obnehin noch nie erstorben, also mit der Irren aufgewachsen ist. Als sie vor 2 Jahren unbäßlich war, wirkten selbst die heftigsten Drastika erst dann, als ihr die Medizin von einem alten geistlichen Herrn benediktirt worden war. "32 Um den Zustand Johanna R.'s „[...] zu heben wurde von Seite der Eltern zwar kein ärztlicher Beistand eingeholt, allein sie suchten Rath und Hülfe bei der Geistlichkeit." Johanna ging daraufhin nicht nur oft freiwillig beichten, sondern wurde laut der Krankengeschichte auch manchmal von ihrer ,[...] vielleicht $z u$ strengen Mutter [...]" zur Beichte gezwungen. Die Handlungsweise der Mutter überrascht nicht, wurde doch über sie angegeben, dass sie vor Jahren von einem Irrsinn befallen worden war, der aber , [...] blo $\beta$ durch Tröstung, Belehrung und geistlichen Zuspruch [...] “ verschwunden sei. Der Beichtvater belegte Johanna mit strengen Bußen, wodurch laut Arzt „[...] ihr ohnehin schon gestörter Gemüths- und Geisteszustand nur noch mehr zerrüttet werden mußte. “33

Bei Exorzismen galt die Vorschrift, Besessene über Gaukeleien und Schwächen der Dämonen zu belehren. Neben den öffentlichen Gebeten könne es auch hilfreich sein zu fasten, da die Dämonen vor allem an Völlerei und Müßiggang Gefallen finden würden. ${ }^{34}$ Insofern stellte sich dieses Bild, dass Dämonen zu Müßiggang verleiteten und dadurch der Zustand für andere wahrnehmbar wurde, neben die ärztliche Erklärung, dass sich Müßigkeit und „unmoralischer Lebenswandel“ als starke Reize auf das Nervensystem auswirkten und das Individuum noch stärker für die Krankheit disponierten.

Nicht erkrankte Angehörige wie ebenso Geistliche förderten häufig eine geistliche Behandlung. So schrieb der Arzt über die Bauerntochter

32 F16, Krankengeschichte 1840.

33 F11, Krankengeschichte 1835.

34 JÜTTE, Geschichte 84. 
Josefa S., dass aus der fortwährenden Furcht vor ewiger Verdammung notwendig auch die Phantasie erkrankt sei, so dass sie mit dem Teufel sprach und aus jedem noch so unbedeutenden Ereignis eine Beziehung auf sich ableitete „[...] und bei dieser somit verkehrten Richtung der Phantasie werden dann auch natürlich verkehrte Mittel zur Beseitigung dieser Trugbilder angewendet. ${ }^{\text {"35 }}$ Sie selber erzählte dazu bei ihrer Aufnahme in Hall, dass ein Pfarrer sie öfter benediziert und versucht habe, den Teufel auszutreiben. Als das aber nicht half, habe er sie zu diesem Zweck zu einem Bischof geschickt, der vom Teufel jedoch nichts wissen wollte und sie an ärztliche Hilfe verwies. ${ }^{36}$ Dazu beschrieb sie die genaue Empfindung, wie der Teufel vom Kopf in sie hinein fuhr und über die Ferse wieder entwich: „Über ihre Empfindungen äußerte sie sich immer mit einem Strome von Worten, es beginne dieses zusammenziehende, spannende Gefühl bald am Unterleibe, und steige dann von hier gegen die Brust und den Hals, den es ihr beynahe ganz zusammenschnüre, und komme dann in den Kopf, von wo es sich auf die Hände verbreitet, es sey gerade als wenn ein lebendes Thier sich in ibr befinde, und dies sey der böse Feind, sie verlangt stets, daß unser $H$. Caplan sie benedicire, denn sie habe schon öfters bemerkt, als jener Pfarrer sie benedicirte, daß der Teufel vom Kopfe hineingefahren, und durch die Ferse von ibr gewichen sey. "37

\section{Zuschreibung negativer Eigenschaften}

Die Bezeichnung der Erkrankten als „Arme“ und „Unglückliche“ wie auch als „Kranke“ und „Patienten“ reflektiert in beiden Vorstellungssystemen den Opferstatus der betroffenen Menschen. Darunter kommt jedoch durch besonders abfällige Beschreibungen und Zuschreibungen von negativen Eigenschaften wie Tücke, Boshaftigkeit und Rache die Wahrnehmung im alltäglichen Umgang mit psychisch Kranken und vielleicht das eigene Distanzierungsbedürfnis der nicht erkrankten Laien und Ärzte zum Vorschein.

Ein zunächst vermuteter Zusammenhang mit der Zuschreibung eines ausschweifenden Lebenswandels ließ sich nicht belegen. Stattdessen zeigten sich andere Tendenzen. Von den zwei Frauen (Tab. 1.1) und vier Männern (Tab. 1.2) handelte es sich in vier Fällen um Personen, deren Krankheit im Verständnis der Zeit - schon bei der Aufnahme diagnostiziert oder später angemerkt - in „Blödsinn“ übergangen war. Über die Bauerntochter Kreszenz S. schrieb Sekundararzt Gottfried Vorhauser beispielsweise: „Ein höchst läppisches, träges Wesen, eine zusammengedrängte, vierschrötige Figur, ganz vegetativ - nur niedere physische Triebe - Verstandsnullität, einseitiges, abgeschmacktes Phantasiespiel - entsprechend dumme Willensäußerung - läppisch 
grobes Maulen - einfältiges Gelächter-Gefräßigkeit, gute Verdauung $u$. Fettwerden sind die auszeichnenden Hauptzüge dieser Patientinn geblieben. "38 Sekundararzt Isidor Mörz bezeichnete Viktoria P. als immer dieselbe „[...] Schaafs-Miene [...] "39 und Primararzt Johann Tschallener meinte über sie: „Sie ist und bleibt Geistes-Krüppel.“40 Die Schafmetapher scheint auf Frauen öfter angewandt worden sein. Zwar nicht als besonders abwertend auffallend, aber der Ergänzung halber hier angefügt, schrieb der Arzt über Theres E. beispielsweise, dass sie „[...] einem verlobrenen Schaaf ähnlich [... $]^{\text {"41 }}$ herumirre. Das Pendant in der Beschreibung von Männern, vor allem, wenn sie häufig onanierten, lag im Bild des Bockes wie bei Thomas R.: „Sein Hinken dürfte wohl auch von seiner lasterhaften Onanie, die er früher ärgerlich und abschreckend getrieben haben soll, erklärbar sein, denn die Böcke in ihrem herabgekommenen Zustand haben alle eine mehr gebückte Stellung und einen ungelenkigen Gang. “42

Dass psychisch Kranke mehr noch in die Nähe der Tiere als etwa der Kinder gerückt wurden, geht auch aus Tschalleners Beschreibung der Anstalt hervor, der seine Aufgabe darin sah, psychisch Kranke „[...] von der Thierheit zur Menschheit wieder empor [... "“43 $\mathrm{zu}$ heben.

Eine besondere Stellung nahmen in der Wahrnehmung die aufgenommenen Erkrankten jüdischer Religion ein. Der Kaufmann Abraham L. achtete auch in der Anstalt sehr auf die Durchführung der jüdischen Religionsübungen. Dies hob ihn stärker von den übrigen PatientInnen ab, als dies bei den beiden Schwestern Bertha H. und Sofia S. der Fall war. Während bei den Frauen kaum Hinweise auf ihre jüdische Religion erwähnt wurden, handelte es sich bei ihnen auch um Gattinnen einflussreicher Kaufleute, fiel die Beschreibung Abraham L.'s sehr stark negativ gefärbt aus. Hieß es oft bei anderen PatientInnen, dass er oder sie immer der oder die gleiche Kranke oder auch Irre blieb, so wurde nun geschrieben, dass Abraham L. immer der gleiche Jude oder Schwächling blieb - angereichert noch mit typischen Zuschreibungen an Juden: „In psychischer Beziehung war in den kommenden Monathen Oktober u. November - Dezember fast gar keine vortheilhafte Änderung mehr wahrzunehmen; denn er blieb der unveränderte blödsinnige Schwächling, nur der groß-u. kleinmäklerische Gewinngeist,

38 F15, Ordinationszettel Februar 1840.

39 F6, Ordinationszettel Juli 1839.

40 F6, Ordinationszettel August 1839.

41 F27, Krankengeschichte 1847.

42 M29, Ordinationszettel Juni 1850.

43 Johann TSCHALLENER, Beschreibung der k. k. Provinzial-Irren-Heilanstalt zu Hall in Tirol; mit Rücksicht auf die Statuten der Anstalt, auf die therapeutischen und psychologischen Grundsätze der Behandlung der Geisteskranken und auf ihre achtjährigen Resultate. Mit 19 Krankengeschichten und verschiedenen Andeutungen zum Wohl dieser Unglücklichen; nebst einem Anhange über die Anlage von Zimmern für Irre und Tobende (Innsbruck 1842) 1. 
u. das in dieser Geistes-Nacht noch hervortappende Bewußtseyn des zusammengerafften Reichthumes ließen sich in seinen gehaltlosen ÄuBerungen wahrnehmen." ${ }^{44}$

Während abfällige Bemerkungen über die Kranken vor allem von Ärzten stammten, sprach aus den Beschreibungen der Laien eher die Furcht im Umgang mit psychisch Kranken. Sie nahmen die Erkrankten vielfach als boshaft, tückisch und voller Rachegelüste wahr. Diese Zuschreibungen wurden bei den acht Frauen (Tab. 1.1) und vier Männern (Tab. 1.2) fünf Mal in Laiendokumenten, sieben Mal in den Krankengeschichten und sechs Mal in der Anamnese bzw. den Ordinationszetteln vorgenommen. Hier besteht auch ein Zusammenhang mit der Gruppe der Erkrankten, die aufgrund ihres beschriebenen ausschweifenden Lebenswandels in der ersten Entstehungskategorie zusammengefasst wurden, indem es sich bei den insgesamt zwölf Fällen sieben Mal um Personen aus dieser ersten Gruppe handelt.

Der Gemeindekassier Johann H. bat 1831 beispielsweise im Namen der ganzen Gemeinde vor Gericht, ,[...] daß die wahnsinnige und im vollsten Maße boshafte [...]" Barbara G. aus dem Dorf entfernt werde. Sie habe mit einem Hammer, den sie von ihrer ,[...] nicht minder boshaft scheinenden Mutter [... $]^{\text {“45 }}$ bekommen habe, eine Frau an der Stirn verletzt. Die Formulierung dieser Aussage korrespondierte fast wortwörtlich mit dem Begleitschreiben des Distriktsarztes zur Krankengeschichte, welche Dokumente der Gemeindekassier als Bestätigung vorlegte. Der Pfarrer betonte den „[...] unmoralischen Lebenswandel [...]" der Kranken und stellte in der Folge die Erkrankung in Frage: „Uebrigens wird dieselbe als eine schlaue, durchtriebene Person geschildert, daher bey einigen sogar der Verdacht rege ward; ihr gegenwärtiger Wahnsinn dürfte, so nicht ganz doch zum Theile, auf Verstellung beruhen. ${ }^{* 46}$

Neben den Bezeichnungen boshaft und tückisch kam auch immer wieder das Motiv der Rache vor. In diesem Zusammenhang musste man sich vor den Kranken schützen. Indem man den Kranken Rache unterstellte, wurde das Zusammenleben natürlich sehr gefährlich und nicht mehr einkalkulierbar. Diese Angst vor Rache resultierte vor allem aus dem Umstand, dass die Erkrankten in ihren Forderungen oder bei tobsüchtigen Anfällen meist in ihrer körperlichen Beweglichkeit eingeschränkt wurden. Sebastian U. war nach zwei Jahren Zuchthaus mit einem Schreiben des Landgerichts an die zuständige Gemeindevorstehung entlassen worden, worin stand, ,[...] daß er sich während seiner Strafzeit in ein an Unsinn gränzendes Geschwätz geredet habe, daß er im Ganzen ein verdorbener, irreligiöser, eigensinniger, leidenschäft-

44 M14, Anamnese 1838.

45 F5, Gerichtsprotokoll 1831.

46 F5, Bericht des Pfarrers 1831. 
licher und Rachsüchtiger Mensch sey, der wegen seiner Rachsucht auch wirklich als Gefährlicher schien und man hat eben deßwegen, strenge Aufsicht auf ihn haben anbefoblen. " ${ }^{47}$

Die Beschreibungen der Erkrankten spiegelten also die Schwierigkeiten des Umgangs mit ihnen wider. Angst spielte für die Ärzte eine geringere Rolle, da sie die Machtmittel zur Beschränkung und Bestrafung in der Hand hatten. Abfällige Bewertungen schienen einerseits aus der Haltung gegenüber den als nicht mehr heilbar diagnostizierten Kranken und andererseits gegenüber abweichenden religiösen Glaubensmeinungen zu resultieren.

\section{Entstehungserklärungen von Geisteskrankheit}

Ausgehend von den Erklärungen der Angehörigen und Bekannten, die sich aus der Krankengeschichte bzw. Berichten von Angehörigen, Geistlichen und Gerichtsprotokollen herausfiltern lassen und die somit als Deutungsangebot der Laien verstanden werden, zeichneten sich drei Erklärungsmuster ab. Bei den ersten beiden Gruppen handelte es sich um Abweichungen von den üblichen Verhaltensnormen. Ein als ausschweifend bezeichneter Lebenswandel, den man verurteilte, wurde ebenso wie ein verstärkter Hang zur Einsamkeit und eine als übertrieben empfundene Ausübung der religiösen Pflichten als Auffälligkeit beschrieben. Trotzdem brachte man - besonders in der zweiten Gruppe - das Auftreten von psychischer Krankheit in engen Zusammenhang mit auslösenden Ereignissen. Die überwiegende Mehrheit der Fälle ließ sich einem dritten Muster zuordnen - dem überraschenden Auftreten von psychischer Krankheit. Dieser Umstand passt zu dem Wahrnehmungsmodell, dass eine psychische Krankheit den Menschen plötzlich befällt.

Bei den 60 Frauen und Männern der Stichprobe wurde in 47 Fällen ein verursachendes Ereignis angegeben (Tab. 2.1. und 2.2.). Als solche Ereignisse kamen bei den Frauen in absteigender Folge der Nennungen vor: missglückte Liebe (8), Abwesenheit, Krankheit oder Tod einer nahe stehenden Person (5), Schwangerschaften und Geburten (4), Menstruation (3), Streit und Familienzwist (2), Schreckerlebnisse (2), nicht erfüllter Berufswunsch (2), berufliche Belastungen (1) und eigene Krankheiten (1). Bei den Männern waren dies: berufliche Belastungen (7), Streit und Familienzwist (4), eigene Krankheiten (4), Abwesenheit, Krankheit oder Tod einer nahe stehenden Person (4), missglückte Liebe (2) und Schreckerlebnisse (2). In drei Fällen wurden zwei verursachende Ereignisse genannt, die sich auf zwei Erkrankungen hintereinander bezogen. 
Diese Angaben wurden teilweise schon von den Ärzten und Wundärzten, die die Krankengeschichte erstellten, spätestens aber von den Ärzten in der Anstalt mit einer zusätzlichen Interpretation versehen. Innerhalb des ärztlichen Modells der disponierenden Ursachen, die nur eines leichten auslösenden Reizes bedürften, nahmen die angegebenen Ereignisse nur einen sehr geringen Erklärungswert ein. Dies lässt sich an den Tabellen 2.1. und 2.2. ablesen, indem die Distrikts- und Kreisärzte in der Krankengeschichte dem angegebenen Ereignis in der Regel noch etwas an Gewicht zumaßen, die Ärzte in der Anstalt jedoch kaum mehr. Eine Ausnahme bilden vier Nennungen von auslösenden Ereignissen in der Anamnese bzw. auf den Ordinationszetteln bei den Männern (Tab. 2.2.). Dabei handelte es sich jeweils um Krankheiten, die sowohl von den Laien als auch von den Ärzten als schwerwiegende Ursache betrachtet wurden.

Aus diesen Tabellen ist auch in den drei Spalten der Erklärungsmuster ersichtlich, dass die Ärzte in Hall mit den Laien insofern übereinstimmten, als sie ausschweifenden Lebenswandel und Eigenschaften wie Zurückgezogenheit und das häufige Lesen von geistlichen Büchern als problematisch sahen. Während Laien allerdings diese Beobachtungen zusätzlich zu einem verursachenden Ereignis angaben, wurden diese Verhaltensweisen bei den Ärzten zu Ursachen, die bedeutender als das Ereignis gesehen wurden. Folgerichtig stimmten auch die Ursachenerklärungen der Ärzte nicht mit der Wahrnehmung der Laien überein, dass das Auftreten der Krankheit plötzlich aus heiterem Himmel habe erfolgen können.

Im Folgenden geht es immer darum, wie die Ärzte die ihnen gelieferten Angaben in ihr Erklärungsmodell einbauten, welche Schlussfolgerungen sie zogen und wo sie mit den Erklärungen der Laien übereinstimmten.

\section{Beschreibung eines ausschweifenden Lebenswandels}

Da von der Stichprobe sieben Frauen (Tab. 2.1) und acht Männer (Tab. 2.2) dieser ersten Gruppe zugeordnet werden, lässt sich aufgrund der Quantität kein geschlechtsspezifischer Unterschied ausmachen. Von den 13 Personen, die für die BeobachterInnen ohne ein spezielles Ereignis erkrankt waren, ließen sich acht Fälle dieser Kategorie zuweisen.

In diesem Muster wurden von Angehörigen, öfter aber von Nachbarn, Geistlichen und Dorfbewohnern Angaben zu einem abgelehnten, verwerflichen Lebenswandel gemacht. In diese Gruppe gehören verstärkt auch die Erkrankten, denen Boshaftigkeit und Tücke zugeschrieben wurde. Die Umgebung bemerkte, dass sich jemand nicht den Normen gemäß verhielt. Festgemacht wurde dies vor allem an der nicht mehr vorhandenen Bereitschaft zur Arbeit und an dem als zu frei empfunde- 


\begin{tabular}{|c|c|c|c|c|c|c|c|c|c|c|c|c|c|c|}
\hline & $\begin{array}{l}\text { Soziale } \\
\text { Herkunft }\end{array}$ & $\begin{array}{l}1 . \mathrm{E} \\
\text { Bes } \\
\text { ei } \\
\text { sch } \\
\text { Leb }\end{array}$ & $\begin{array}{l}\text { rklär } \\
\text { nuste } \\
\text { chreil } \\
\text { nes a } \\
\text { weife } \\
\text { nswa }\end{array}$ & $\begin{array}{l}\text { ngs- } \\
\vdots \\
\text { ung } \\
\text { s- } \\
\text { den } \\
\text { adels }\end{array}$ & $\begin{array}{l}\underline{2 . \mathrm{E}} \\
\text { mu } \\
\text { zur } \\
\text { und } \\
\text { ben } \\
\text { ons }\end{array}$ & $\begin{array}{l}\text { rklär } \\
\text { ter: } \\
\text { Einsa } \\
\text { „übe } \\
\text { e“ Re } \\
\text { ausül }\end{array}$ & $\begin{array}{l}\text { ngs- } \\
\text { ang } \\
\text { akeit } \\
\text { trie- } \\
\text { ligi- } \\
\text { ung }\end{array}$ & \begin{tabular}{|r} 
3. E \\
Über \\
Auf \\
psy \\
Kr
\end{tabular} & $\begin{array}{l}\text { rkläru } \\
\text { nuster } \\
\text { rasch } \\
\text { treten } \\
\text { chisc } \\
\text { ankhe }\end{array}$ & $\begin{array}{l}\text { ings- } \\
\text { r: } \\
\text { endes } \\
\text { von } \\
\text { hen } \\
\text { iten }\end{array}$ & $\begin{array}{l}\text { Angabe eines } \\
\text { verursachenden } \\
\text { Ereignisses }\end{array}$ & $\begin{array}{r}\text { Bew } \\
\text { veru } \\
\text { Er }\end{array}$ & $\begin{array}{l}\text { Starke } \\
\text { ertung } \\
\text { rsache } \\
\text { eignis }\end{array}$ & $\begin{array}{l}\text { des } \\
\text { nden } \\
\text { ses }\end{array}$ \\
\hline & & LD & KG & $\begin{array}{l}\text { A/ } \\
\mathrm{OZ}\end{array}$ & LD & KG & $\begin{array}{l}\text { A/ } \\
\mathrm{OZ}\end{array}$ & LD & KG & \begin{tabular}{|c|} 
A/ \\
$\mathrm{OZ}$ \\
\end{tabular} & & LD & KG & $\begin{array}{l}\mathrm{A} / \\
\mathrm{OZ} \\
\end{array}$ \\
\hline F1 & \begin{tabular}{|c|} 
Gattin eines \\
Handelsmanns \\
\end{tabular} & & & & & & & & $\mathbf{x}$ & & $\mathbf{x}$ & & & \\
\hline $\mathrm{F} 2$ & $\begin{array}{l}\text { Schmieds- } \\
\text { tochter }\end{array}$ & & & & & $\mathbf{x}$ & $\mathbf{x}$ & & & & $\mathbf{x}$ & & $\mathbf{x}$ & \\
\hline F3 & $\begin{array}{l}\text { Schicht-mei- } \\
\text { sterstochter }\end{array}$ & & & & & & & & $\mathbf{x}$ & & $\mathbf{x}$ & & $\mathbf{x}$ & \\
\hline F4 & $\begin{array}{l}\text { Gattin eines } \\
\text { Polizei- } \\
\text { kommissärs }\end{array}$ & & & & $\mathbf{x}$ & $\mathrm{x}$ & $\mathbf{x}$ & & & & $\mathbf{x}$ & & & \\
\hline F5 & Magd & $\mathbf{x}$ & $\mathbf{x}$ & $\mathbf{x}$ & & & & & & & & & & \\
\hline F6 & Magd & & & & & & & & $\mathbf{x}$ & & $\mathbf{x}$ & & $\mathbf{x}$ & \\
\hline F7 & Magd & & & & & & & & $\mathbf{x}$ & & $\mathbf{x}$ & & $\mathbf{x}$ & \\
\hline F8 & Bäuerin & & & & & & & & $\mathbf{x}$ & & $\mathbf{x}$ & & $\mathbf{x}$ & \\
\hline F9 & \begin{tabular}{c|} 
Gattin eines \\
Handelsmanns \\
\end{tabular} & & & & & & & & $\mathbf{x}$ & & $\mathbf{x}$ & & $\mathbf{x}$ & \\
\hline F10 & $\begin{array}{c}\text { Bettelei / } \\
\text { Dienstmagd }\end{array}$ & & $\mathbf{x}$ & $\mathbf{x}$ & & & & & & & & & & \\
\hline F11 & Bauerntochter & & & & & $\mathbf{x}$ & $\mathbf{x}$ & & & & $\mathbf{x}$ & & $\mathbf{x}$ & \\
\hline F12 & Tagelöhnerin & & & & & & & & $\mathbf{x}$ & & & & & \\
\hline F13 & $\begin{array}{l}\text { Gattin eines } \\
\text { Kanzlisten }\end{array}$ & & & & & & & & $\mathbf{x}$ & & $\mathbf{x}$ & & $\mathbf{x}$ & \\
\hline F14 & Bäuerin & & & & & & & & $\mathbf{x}$ & & $\mathbf{x}$ & & $\mathbf{x}$ & \\
\hline F15 & Bauerntochter & & & & & & & & $\mathbf{x}$ & & $\mathbf{x}$ & & $\mathbf{x}$ & \\
\hline F16 & Bauerntochter & & $\mathbf{x}$ & $\mathbf{x}$ & & & & & & & $\mathbf{x}$ & $\mathbf{x}$ & $\mathbf{x}$ & \\
\hline F17 & Bauerntochter & & & & & & & & $\mathbf{x}$ & & $\mathbf{x}$ & & $\mathbf{x}$ & \\
\hline F18 & Tagelöhnerin & $\mathbf{x}$ & $\mathbf{x}$ & $\mathbf{x}$ & & & & & & & $\mathbf{x}$ & $\mathbf{x}$ & $\mathbf{x}$ & \\
\hline F19 & Magd & & & & & $\mathbf{x}$ & $\mathbf{x}$ & & & & $\mathbf{x}$ & & $\mathbf{x}$ & \\
\hline F20 & \begin{tabular}{|c|} 
Gattin eines \\
Sattlermeisters \\
\end{tabular} & & & & & & & & $\mathbf{x}$ & & $\mathbf{x}$ & & $\mathbf{x}$ & \\
\hline F21 & Güterbesitzerin & & & & & & & & $\mathbf{x}$ & & $\mathbf{x}$ & & $\mathbf{x}$ & \\
\hline F22 & Bauerntochter & & & & & $\mathbf{x}$ & $\mathbf{x}$ & & & & $\mathbf{x}$ & & $\mathbf{x}$ & \\
\hline F23 & Magd & & $\mathbf{x}$ & $\mathbf{x}$ & & & & & & & $\mathbf{x}$ & & $\mathbf{x}$ & \\
\hline F24 & $\begin{array}{l}\text { Witwe eines } \\
\text { Landrichters }\end{array}$ & & $\mathbf{x}$ & $\mathbf{x}$ & & & & & & & & & & \\
\hline F25 & Magd & & & & & $\mathbf{x}$ & $\mathbf{x}$ & & & & $\mathbf{x}$ & & $\mathbf{x}$ & \\
\hline F26 & Wirtschafterin & & & & & & & & $\mathbf{x}$ & & $\mathbf{x}$ & & $\mathbf{x}$ & \\
\hline F27 & Bauerntochter & & $\mathbf{x}$ & & & & & & & & $\mathbf{x}$ & & $\mathbf{x}$ & \\
\hline F28 & Bauerntochter & & & & & $\mathbf{x}$ & $\mathbf{x}$ & & & & $\mathbf{x}$ & & $\mathbf{x}$ & \\
\hline F29 & $\begin{array}{l}\text { Handlungs- } \\
\text { besitzerin }\end{array}$ & & & & & & & & $\mathbf{x}$ & & $\mathbf{x}$ & & $\mathbf{x}$ & \\
\hline F30 & Kammerjungfer & & & & & & & & $\mathbf{x}$ & & $\mathbf{x}$ & & $\mathbf{x}$ & \\
\hline & Summen & 2 & 7 & 6 & 1 & 7 & 7 & 3 & 16 & 0 & 26 & 2 & 24 & 0 \\
\hline
\end{tabular}

Tab. 2.1. Entstehung von psychischer Krankheit bei Frauen (Krankenakten mit Laiendokumenten grau unterlegt) 


\begin{tabular}{|c|c|c|c|c|c|c|c|c|c|c|c|c|c|c|}
\hline & \multirow[t]{2}{*}{$\begin{array}{l}\text { Soziale } \\
\text { Herkunft }\end{array}$} & \multicolumn{3}{|c|}{$\begin{array}{l}\text { 1. Erklärungs- } \\
\text { muster: } \\
\text { Beschreibung } \\
\text { eines aus- } \\
\text { schweifenden } \\
\text { Lebenswandels }\end{array}$} & \multicolumn{3}{|c|}{$\begin{array}{l}\text { 2. Erklärungs- } \\
\text { muster: Hang } \\
\text { zur Einsamkeit } \\
\text { und „übertrie- } \\
\text { bene“ Religi- } \\
\text { onsausübung }\end{array}$} & \multicolumn{3}{|c|}{$\begin{array}{l}\text { 3. Erklärungs- } \\
\text { muster: } \\
\text { Überraschendes } \\
\text { Auftreten von } \\
\text { psychischen } \\
\text { Krankheiten }\end{array}$} & \multirow[t]{2}{*}{$\begin{array}{c}\text { Angabe eines } \\
\text { verursachenden } \\
\text { Ereignisses }\end{array}$} & \multicolumn{3}{|c|}{$\begin{array}{c}\text { Starke } \\
\text { Bewertung des } \\
\text { verursachenden } \\
\text { Ereignisses }\end{array}$} \\
\hline & & LD & KG & $\begin{array}{l}\mathrm{A} / \\
\mathrm{OZ}\end{array}$ & LD & KG & $\begin{array}{l}\mathrm{A} / \\
\mathrm{OZ}\end{array}$ & LD & KG & $\begin{array}{l}\mathrm{A} / \\
\mathrm{OZ}\end{array}$ & & LD & KG & $\begin{array}{l}\mathrm{A} / \\
\mathrm{OZ}\end{array}$ \\
\hline M1 & Sattlergeselle & & & & & & & & $\mathbf{x}$ & & $\mathbf{x}$ & & $\mathbf{x}$ & $\mathbf{x}$ \\
\hline M2 & $\begin{array}{l}\text { Ehemaliger } \\
\text { Lieutenant }\end{array}$ & & & & & & & $\mathbf{x}$ & $\mathbf{x}$ & & $\mathbf{x}$ & $\mathbf{x}$ & $\mathbf{x}$ & \\
\hline M3 & Knecht & & & & & & & & $\mathbf{x}$ & & & & & \\
\hline M4 & Knecht & & & & & & & & $\mathbf{x}$ & & $\mathbf{x}$ & & $\mathbf{x}$ & \\
\hline M5 & $\begin{array}{c}\text { Rotgerber- } \\
\text { geselle }\end{array}$ & & & & & & & & $\mathbf{x}$ & & $\mathbf{x}$ & & & \\
\hline M6 & Knecht & & & & & & & & $\mathbf{x}$ & & $\mathbf{x}$ & & $\mathbf{x}$ & $\mathbf{x}$ \\
\hline M7 & Kleinbauer & $\mathbf{x}$ & & $\mathbf{x}$ & & & & & & & & & & \\
\hline M8 & Wegner & & & & & & & & $\mathbf{x}$ & & $\mathbf{x}$ & & $\mathbf{x}$ & \\
\hline M9 & Bäcker & & & & & & & & $\mathbf{x}$ & & $\mathbf{x}$ & & $\mathbf{x}$ & $\mathbf{x}$ \\
\hline M10 & Tagelöhnersohn & & & & & & & & $\mathbf{x}$ & & $\mathbf{x}$ & & $\mathbf{x}$ & \\
\hline M11 & Förster & & $\mathbf{x}$ & $\mathbf{x}$ & & & & & & & $\mathbf{x}$ & & $\mathbf{x}$ & \\
\hline M12 & Wirt & & $\mathbf{x}$ & $\mathbf{x}$ & & & & & & & & & & \\
\hline M13 & Bauernsohn & & & & & & & & $\mathbf{x}$ & & & & & \\
\hline M14 & Kaufmann & & & & & & & & $\mathbf{x}$ & & $\mathbf{x}$ & & $\mathbf{x}$ & \\
\hline M15 & Weber & & & & & $\mathbf{x}$ & $\mathbf{x}$ & & & & $\mathbf{x}$ & & $\mathbf{x}$ & \\
\hline M16 & $\begin{array}{l}\text { Hauptmann } \\
\text { Kaiserjäg.reg. }\end{array}$ & & & & & & & & $\mathbf{x}$ & & $\mathbf{x}$ & & $\mathbf{x}$ & \\
\hline M17 & Priester & & & & & & & & $\mathbf{x}$ & & $\mathbf{x}$ & & $\mathbf{x}$ & \\
\hline M18 & Maurer & & $\mathbf{x}$ & & & & & & & & & & & \\
\hline M19 & $\begin{array}{l}\text { Stellwagen- } \\
\text { inhaber }\end{array}$ & & $\mathbf{x}$ & $\mathbf{x}$ & & & & & & & $\mathbf{x}$ & & $\mathbf{x}$ & \\
\hline M20 & Wundarzt & & & & & & & $\mathbf{x}$ & $\mathbf{x}$ & & $\mathbf{x}$ & $\mathbf{x}$ & $\mathbf{x}$ & \\
\hline M21 & Kanzlist & & & & & & & & $\mathbf{x}$ & & & & & \\
\hline M22 & Doktorand & & & & & & & & $\mathbf{x}$ & & $\mathbf{x}$ & & $\mathbf{x}$ & \\
\hline M23 & $\begin{array}{l}\text { Ehemaliger } \\
\text { Theologe }\end{array}$ & & & & & $\mathbf{x}$ & $\mathbf{x}$ & & & & & & & \\
\hline M24 & Bergknappe & & & & & $\mathbf{x}$ & $\mathbf{x}$ & & & & $\mathbf{x}$ & & $\mathbf{x}$ & $\mathbf{x}$ \\
\hline M25 & Bauer & & & & & $\mathbf{x}$ & $\mathbf{x}$ & & & & $\mathbf{x}$ & & & \\
\hline M26 & $\begin{array}{c}\text { Schiffs- } \\
\text { zimmermann }\end{array}$ & & & & & & & & $\mathbf{x}$ & & $\mathbf{x}$ & & $\mathbf{x}$ & \\
\hline M27 & $\begin{array}{l}\text { Müller und } \\
\text { Musikus }\end{array}$ & & & & & & & & $\mathbf{x}$ & & $\mathbf{x}$ & & $\mathbf{x}$ & \\
\hline M28 & $\begin{array}{c}\text { Finanzwächter/ } \\
\text { Zuchthaus }\end{array}$ & $\mathbf{x}$ & $\mathbf{x}$ & $\mathbf{x}$ & & & & & & & & & & \\
\hline M29 & Schuhmacher & & $\mathbf{x}$ & $\mathbf{x}$ & & & & & & & & & & \\
\hline \multirow[t]{2}{*}{ M30 } & Wirtssohn & & $\mathbf{x}$ & $\mathbf{x}$ & & & & & & & $\mathbf{x}$ & & & \\
\hline & Summen & 2 & 7 & 7 & 0 & 4 & 4 & 2 & 28 & 0 & 21 & 2 & 18 & 4 \\
\hline
\end{tabular}

Tab. 2.2. Entstehung von psychischer Krankheit bei Männern (Krankenakten mit Laiendokumenten grau unterlegt) 
nen Umgang mit dem anderen Geschlecht. Beides ging meist Hand in Hand. Da für die Bevölkerung der Beginn einer psychischen Krankheit ganz eng mit Ereignissen verknüpft war, tendierte sie dazu, bei Mangel eines solchen Ereignisses den Erkrankten Verstellung zuzuschreiben. Selbst bei Anerkennung einer Krankheit verschwammen sehr oft die Grenzen, ab wann zum Beispiel so genanntes arbeitsscheues Verhalten als Eigenschaft des Menschen und wann als Symptom der Krankheit gesehen wurde.

Wir haben es hier zunächst mit Mägden, Tagelöhnerinnen und Bettlerinnen zu tun, die wie beispielsweise Barbara G. als „[...] seit Jahren roh, ausgelassen in ibren Sitten; arbeitsscheu; dem Bettel ergeben; selbst, wie die Rede geht, unerlaubten Erwerb nicht verschmähend [...] aller Frömmigkeit $u$. religiösen Sinne ganz entfremdet, so daß selbe selbst die Pflicht der österlichen Beicht $u$. Kommunion bereits zwey Jahre vernachläßiget habe [... “" ${ }^{48}$ beschrieben wurden. Das Vorhandensein einer Krankheit wurde vom Pfarrer in Frage gestellt und vielmehr Verstellung aufgrund ihrer Boshaftigkeit vermutet.

Es handelte sich hierbei um Frauen, die aufgrund von Umständen, die nicht weiter thematisiert wurden, den dörflichen Normen von einem arbeitsamen Erwerbsleben und einer geregelten Sexualität nicht entsprachen oder nicht entsprechen konnten. Das Wort „leichtsinnig“ deutet immer auf einen zugeschriebenen lockeren Umgang mit dem anderen Geschlecht, ${ }^{49}$ wie auch der Zusammenhang, sich früh der physischen Liebe hingegeben zu haben und uneheliche Kinder bekommen zu haben. Beides bekommt allerdings ein anderes Gewicht vor den rechtlichen Heiratsbeschränkungen der Zeit. ${ }^{50}$

Betrachtet man nun die Männer, die in diese Kategorie eingeordnet werden können, fällt zunächst ins Auge, dass in der Hälfte der Fälle ein plötzlicher Bruch von heiteren, zufriedenen Jünglingsjahren zu einem angeblich beobachteten unmoralischen Lebenswandel thematisiert wurde. Mit Sebastian U. waren bis zu seinem 19. Lebensjahr laut Bericht des Pfarrers seine Eltern und Schullehrer recht zufrieden. Er habe sogar „[...] eine Liebe zum Lesen gezeigt und mit guten Büchern sich oft unterhalten. "Im Jünglingsalter aber begann er sich zu ändern und wurde „[...] eigensinnig, widerspenstig, pflog Umgang mit dem weiblichen Geschlechte und hat sich bald eine natürliche Nachkommenschaft erworben." Auch mit seiner Frau „[...] war er hart, auch hatte er mit einem andern Weibe einen vertrauten Umgang. " 51

48 F5, Bericht des Pfarrers 1831.

49 Vgl. Stefan BREIT, „Leichtfertigkeit“ und ländliche Gesellschaft. Voreheliche Sexualität in der frühen Neuzeit (München 1991).

$50 \mathrm{Vgl}$. Elisabeth MANTL, Heirat als Privileg. Obrigkeitliche Heiratsbeschränkungen in Tirol und Vorarlberg 1820 bis 1920 (Wien 1997).

51 M7, Bericht Pfarrer 1830. 
Daneben gab es auch Beschreibungen, die keine heiteren Knaben- und Jünglingsjahre anführten, weil man entweder zu wenig über sie wusste oder die Erziehung als schlecht beurteilt wurde. Dabei handelte es sich zum Teil um Männer, die bereits abgestraft worden waren. Lediglich Joseph H. wurde als Opfer geschildert, da er schon als Knabe mit „[...] unmäßigen rohen Leuten [...] “ in Berührung kam, die ihn oft ins Wirtshaus mitnahmen und ihm zu trinken gaben, bis er berauscht war. Nach und nach habe er angefangen, „[...] dieses Getränk lieb zu gewinnen, er trank davon mehr, ertrug immer mehr, und bald war er ein perfekter Branntweinsäufer, der Branntwein verfeblte auch nicht seine üblen Folgen [...] Er wurde immer blöder im nüchternen Zustand, im berauschten immer wilder $u$. tobender. " 52 Daraufhin vernachlässigte er bald den Besuch der Kirche, achtete die Religion, in der er erzogen worden war, nicht mehr und vermied den Gebrauch der Sakramente.

Trunksucht und Geschlechtskrankheiten dienten einerseits als Indikatoren eines ausschweifenden Lebens und andererseits als zusätzliche Faktoren in der Entstehung der psychischen Krankheit. Während der Sekundararzt Alois Salcher in Roman K.'s großem Hang zum tieferen Nachdenken und zum Grübeln über verschiedene Gegenstände, worüber alles andere „hintangesetzt“ wurde und er sich, „[...] um frey seinen Lieblingsgedanken nachhängen zu können immer mehr und mehr von seiner gewöhnlichen Gesellschaft, wo er ohne dieß nur Verdrießlichkeiten fand [...]" entfernte und sich zurückzog, eine disponierende Ursache sah, zu der der ununterbrochene Genuss von berauschenden Getränken eine bedeutende Expansion des Blutes und insbesondere Kongestionen zu „[...] den schon durch vieles Meditieren und Anstrengung gereitzten Gehirn [... ${ }^{\text {"53 }}$ bewirkte, sah Tschallener in einer zweiten Version der Anamnese einen anderen Sachverhalt als ausschlaggebend an. Aus Eifersucht habe Roman K. begonnen zu trinken und aus dem Trinken sei die Geisteskrankheit entstanden. ${ }^{54}$

\section{Hang zur Einsamkeit und „übertriebenen“ Religionsausübung}

Auf eine andere Weise nicht der Norm entsprechend waren ein Hang zur Einsamkeit, Zurückgezogenheit, oder wenn sich jemand besonders ängstlich oder religiös verhielt.

Von den 60 Fällen traf diese Zuordnung nur auf sieben Frauen und vier Männer zu (Tab. 2.1. und 2.2.). Da dieses Verhalten oft mit dem Lesen von Büchern verbunden war, könnte eine Erklärung der leichten Mehrheit der Frauen die höhere Verbreitung der Lese- und Schreibfähigkeit unter Frauen sein. Die Bauerntochter Johanna R. zeigte sich mitunter fröhlich und heiter, jedoch war ein Hang zur Einsamkeit 
vorherrschend, „[...] weßhalb sie oft abgeschieden auf ihrer Kammer verweilte, während ihre Geschwisterte in müßigen Stunden am Heimgarth sich ergötzten. "55

Der Bauer Thomas B. habe sich als Jüngling viel mit Lesen von Büchern, insbesondere solcher mit religiösem Inhalt beschäftigt, sich von der Unterhaltung und dem Umgang anderer junger Leute fern gehalten und habe in Zurückgezogenheit und Ruhe gelebt. Jedoch habe er schon in diesen Jahren großen Eigensinn, Rechthaberei und bei jedem Widerspruch auffallenden Trotz gezeigt. ${ }^{56}$

Trotz dieser angegebenen Informationen über das etwas abweichende Verhalten, brachten die Angehörigen der Erkrankten oder diese selbst die psychischen Krankheiten mit bestimmten Ereignissen in Verbindung und bewerteten sie stärker. Die Ärzte griffen die Beschreibungen der Zurückgezogenheit und Ängstlichkeit auf und banden sie wiederum in ihr Erklärungssystem ein. Diese beschriebenen Eigenschaften sahen sie als Nährboden, auf dem sich psychische Krankheiten sehr leicht entwickeln konnten. „Düsteres Dabinbrüten, Einseitigkeit in der geistigen Richtung $u$. darauf folgendes periodisches Lähmen des Geistes $u$. des Willens [...]" seien „[...] die kranken Früchte, deren Reife dadurch zu Stande kam, daß der Keim auf einen fruchtbaren Boden fiel, denn ein melancholisches Temperament ist wohl am ehesten geeignet, bei gegebener Ursache eine Gemüthsdepression, wie bei P. zu erzeugen [...], "57 fasste Sekundararzt Vorhauser die Entstehung von Gertraud P.'s Krankheit zusammen.

Die angegebenen Ereignisse nahmen in den ärztlichen Erklärungen hingegen einen untergeordneten Rang ein. Das öfter erwähnte Lesen von religiösen Büchern diente beispielsweise als Indiz für eine Überbildung der Geisteskräfte. So wurde bei Notburga K. neben der erblichen Anlage, einer durch Kränklichkeit und Krankheiten reizbaren Konstitution auch die fortwährende Anstrengung ihres Geistes, wodurch sie sich überbildete, angegeben. Dies alles habe ein zartfühlendes, leicht zu kränkendes Wesen vorbereitet und sie zur Seelenkrankheit prädisponiert. Lediglich den Ausschlag gegeben habe eine schwere mit Krämpfen verbundene Entbindung. ${ }^{58}$

\section{Überraschendes Auftreten von psychischer Krankheit}

Viele Angehörige und Bekannte beschrieben das Auftreten der Erkrankung als ein sehr überraschendes Vorkommnis. Vor allem benannten sie ein bestimmtes Ereignis, mit dem für sie die Krankheit begonnen

55 F11, Krankengeschichte 1835.

56 M25, Krankengeschichte 1845.

57 F19, Anamnese 1842.

58 F4, Anamnese 1832. 
hatte. Die geschilderten Fälle ähneln in ihrer Struktur sehr dem Bild, das bei den Männern der ersten Kategorie auftauchte: Die Betroffenen waren meist ordentlich, arbeitsam und heiter, bis plötzlich der Umschwung eintrat „[...] und niemand hätte früher an den einsmaligen Ausbruche des Wahnsinns gedacht. ${ }^{* 59}$

Die Mehrzahl der Fälle entspricht dieser Kategorie - 16 Frauen und 18 Männer (Tab. 2.1 und 2.2). Somit zeigt sich mit diesem Befund am deutlichsten, dass die Laienerklärungen nicht mit den ärztlichen Modellen übereinstimmten: Der Großteil der Erkrankungen wurde als plötzliches Ereignis beschrieben, das von irgendwoher zu kommen schien und dem Menschen zustieß.

Typisch für die medizinische Sichtweise suchten die Ärzte wie schon in den beiden anderen Kategorien nach einer länger vorbereiteten Disposition und bewerteten dementsprechend das Ereignis als weniger bedeutend. Während sie beim ersten Modell den ausschweifenden Lebenswandel als Reiz einbauten, der auf eine erbliche Anlage oder geschwächte Konstitution wirkte und im zweiten Modell im zurückgezogenen, ängstlichen Verhalten bzw. umgedeutet zum melancholischen Temperament den Nähr- und Keimboden für die Ausbildung psychischer Krankheit sahen, mussten sie hier sehr viel stärker Vermutungen heranziehen und Verlegenheitsdiagnosen erstellen.

Eine Möglichkeit der ärztlichen Erklärung war eine Zuschreibung von problematischen Eigenschaften und mangelhafter Erziehung. Auf der Suche nach disponierenden Ursachen sahen sie Eigenschaften, die gar nicht in der Krankengeschichte erwähnt wurden. Die Annahme, dass sie dabei vom Verhalten der PatientInnen in der Anstalt - also in bereits erkranktem Zustand - auf früheres Verhalten zurück schlossen, liegt nahe. Der Wert von 21 Nennungen bei den Frauen, die das Temperament, den Charakter bzw. die Eigenschaften als Ursache für die Erkrankungen sahen, hebt sich deutlich von den anderen Ursachen ab, während diese Kategorie bei den Männern mit 13 Nennungen nur an zweiter Stelle liegt. Ärzte schienen also die Ursache für psychische Krankheiten bei Frauen häufig im Gemüt verankert zu haben.

Der Kurat schrieb beispielsweise über die Magd Theresia L., dass er sie „[...] früher als eines der ordentlichesten, stillsten $u$. bescheidensten [...]" Mädchen seines Seelsorgebezirkes kannte, bis die den Wunsch äußerte, in ein Kloster zu gehen. Als ihr das aufgrund der ungünstigen Vermögensverhältnisse nicht erlaubt wurde, „[...] fing man erst an, ibre Geistes Verwirrung etwas zu beobachten. " ${ }^{60}$ Der Arzt formulierte diese Verhaltensänderung so, dass sie im 24. Lebensjahr „[...] verschiedenartige Gedanken [...] “ bekam, das Klosterleben wünschte

59 M3, Krankengeschichte 1830.

60 F7, Bericht des Kuraten 1833. 
und sich dem Schreiben, dem Nachdenken und Nachsinnen verschiedener abwechselnder Gegenstände ergab, „[...] der Arbeit etwas scheu $[\ldots]^{\text {"61 }}$ wurde und lieber von einem Haus zum anderen herumgehen wollte. Demgegenüber legte der Sekundararzt Salcher in der Anamnese dieses „Nicht-gerne-Tun“ zeitlos über ihre gesamte Arbeitstätigkeit und projizierte diesen Unwillen also zeitlich zurück. In der Folge wurde sie bereits als „[...] die halstörrige und eigensinnige L. [...] “62 bezeichnet.

Bei Männern wurden von Laien häufig Anstrengungen im Beruf oder in der Ausbildung als verursachende Ereignisse ins Spiel gebracht. Indem die Ärzte die Auswirkungen auf das Blut, das sich ausdehnte, wie auch auf das Nervensystem und Gehirn beschrieben, passte dies in ihr Erklärungssystem. Über Johann B. hieß es, dass er als Bäcker von der großen Ofenhitze heftige Kopfschmerzen und zeitweise Sinnesverwirrung bekam. ${ }^{63}$ Dies erschien auch Salcher plausibel. Disponierend seien zwar ,[...] seine starke, energische veritable Constitution und sein sanguinisch cholerisches Temperament [...]", jedoch habe die Hitze eine übermäßige Expansion der Gehirngefäße hervorgerufen, auch das in den Adern enthaltene Blut würde durch die Wärme ausgedehnt „[...] und selbes konnte also am leichtesten zum Gehirn hingleitet werden; durch die Menge und gesteigerte Thätigkeit des Blutes wurden [...] die mit dem Gehirn in Verbindung stehenden Nerven in eine größere Activitaet, welche sich dann durch gewaltsamme Handlungen nach Aussen äusserte, versetzt. Durch das nun aufgehobene, oder viellmehr verstumte Gleichgewicht zwischen Seele und Gehirn erscheinen die äusseren Gegenstände seiner Seele unter falschen Bildern. "64

Belastungen durch Arbeit und Beruf bei Frauen wurden anders thematisiert. Das Bild von einer geringeren Befähigung zu außerhäuslicher Erwerbsarbeit, also außerhalb von landwirtschaftlichen Zusammenhängen (im Rahmen dessen Arbeit als Belastung übrigens nie erwähnt wurde), kam in der ärztlichen Argumentationsweise deutlich zum Ausdruck. So fanden sich in der Krankengeschichte von Kreszenz G. überhaupt keine Hinweise auf Eigenschaften, Verhaltensweisen oder körperliche Ursachen, die sie in den Augen der Ärzte für die Krankheit disponiert haben könnten. Sie arbeitete als Wirtschafterin des Bades Obladis und hatte plötzlich begonnen, davon zu sprechen, dass sie nichts dafür könne und dass sie nicht schuldig sei. Deshalb vermutete der Arzt, der die Krankengeschichte verfasste, dass „[...] ihr die $\beta$ Geschäft, und die damit verbundene Verantwortlichkeit zu schwer $[\ldots]^{\text {“ } 65}$ gewesen sei. Sekundararzt Josef Stolz nahm daraufhin an, dass

61 F7, Krankengeschichte 1832.

62 F7, Anamnese 1835.

63 M9, Krankengeschichte 1833.

64 M9, Anamnese 1836.

65 F26, Krankengeschichte 1846. 
Kreszenz G. eine gewissenhafte und ehrgeizige Person gewesen sein müsse, um mit dieser Verwaltungsstelle betraut zu werden. Da sie dieses Geschäft aber ganz ohne männliche Unterstützung betrieb, sei es nicht unwahrscheinlich, dass sie deshalb in eine „gemütliche " ${ }^{66}$ Verwirrung gefallen sei. Diese Sichtweise korrespondierte mit dem ärztlichen Verständnis der Zeit, indem angenommen wurde, dass so wie „[...] im Kinde und Weibe das vegetative, gangliöse Leben vorherrscht [...] Kinder und Weiber (ebenso) fast ganz nur Gemüth [...] "667 seien.

Die häufigste ärztliche Erklärung für die Entstehung von psychischen Krankheiten bei Männern war körperliche Krankheit. So klagte Peter B. über ein „[...] Kuriösseyn im Kopfe (wodurch Peter B. ein Wirbeln in der Stirngegend ausdrucken will) “. Dieses sei vor zehn Jahren ohne bekannte Ursache entstanden. Daraus leitete der die Krankengeschichte verfassende Arzt die Verminderung seiner Verstandeskräfte her, weshalb er auch seinen Beruf - Sattlerhandwerk - nicht richtig habe lernen können. Diese Unfähigkeit habe Niedergeschlagenheit und Schwermut erzeugt und schließlich „[...] die wirkliche Melancholie. “68

Ein wesentlicher Bestandteil in den weiblichen Krankengeschichten war die Thematisierung der Menstruation und - falls die Frauen Kinder geboren hatten - das ganze Umfeld der Schwangerschaften wie auch des Wochenbetts und bei älteren Frauen die Wahrnehmung etwaiger Anzeichen des nahenden Klimakteriums. Während das Ausbleiben der Menstruation von drei Frauen als verursachendes Ereignis für die Erkrankung gesehen wurde, thematisierten die Ärzte in der Anstalt Menstruation als Ursache nur zweimal. Das überrascht nach der ausführlichen Berichterstattung über die monatlichen Menstruationsvorgänge bei den Patientinnen in der Anstalt. Für die Ärzte stellte die Menstruation eine Möglichkeit zur Erklärung dar, wenn sonst keine Ursachen gefunden wurden. So vermutete zum Beispiel der Sekundararzt Joseph Georg Windbüchler bei Viktoria P., da sich „[...] rücksichtlich der ursächlichen Momente [...] nichts Bestimmtes ausmitteln ließ [...]", dass „[...] ihr Seelenleiden mit der Menstruation in Verbindung $[\ldots]^{\text {"69 }}$ stehe.

Die Zeit der Geschlechtsentwicklung wurde vor allem bei Männern öfter als problematische Zeit thematisiert. „Laut Erfahrung vermögen die Jahre der völligen Geschlechtsentwicklung den Menschen gleich dem Insekt auf eine wunderbare Art umzuwandeln, auch sebr oft Krankheit in Gesundheit, und Gesundheit in Krankheit umzuändern: und ich glaube nicht zu irren, wenn ich vermuthe, daß diese

66 F26, Anamnese 1847.

67 Johann Georg HECHENBERGER, Grundriß zu einem Organon der spezifischen Seelenheilkunst, vorzüglich für die Landpraxis gemeinfaßlich dargestellt (Wien 1841) 15.

68 M1, Krankengeschichte 1830.

69 F6, Anamnese 1832. 
große Metamorphose bei diesem Irren abnorm vor sich gegangen sey, und somit Krankheit hervorgebracht habe. Warum sie aber abnorm war, und worin diese Abnormitaet bestehe? - dieß zu eruiren, will ich mich gerne für unfähig erkennen, [... " ${ }^{* 70}$ schrieb der Arzt über Johann Ach. Jedoch nahmen die Ärzte trotzdem bei Männern nur zweimal eine starke oder falsche Entwicklung des Geschlechtstriebs als disponierende Ursache an. Demgegenüber wurde bei Frauen sechsmal ein falsch entwickelter oder zu früh geweckter Geschlechtstrieb als Ursache angesprochen. Bertha H. habe beispielsweise einen vielleicht , [...] schon früh durch Romane lesen geweckten Geschlechtstrieb, bey der Impotenz ibres Mannes, unerlaubt befriediget und von ihrem Gewissen Vorwürfe erhalten. " 71

Onanie $^{72}$, besonders wenn sie in der Anstalt beobachtet wurde, lieferte den Ärzten eine gute Erklärung für körperliche Veränderungen bzw. die Entstehung von psychischen Krankheiten. Zwar wurde die Onanie durch die Verbindung mit den schädigenden Auswirkungen in die Nähe der körperlichen Krankheiten gerückt, doch schwang in der Beschreibung und der in der Irrenanstalt erfolgenden Bestrafung und Beschränkung durch die Zwangsjacke nach wie vor die Beurteilung als moralisches Vergehen mit. Insgesamt wurde Onanie als Ursache innerhalb der ärztlichen Erklärungen bei den Männern viermal und bei den Frauen dreimal genannt. Als verursachendes Ereignis wurde es von den Laien nie angegeben. Darauf deutet auch der Umstand, dass Selbstbefriedigung vor Einführung einer entsprechenden Frage in den 1835 für die Erfassung der Krankengeschichte genormten Fragebogen in den Krankengeschichten kein Thema war. Jedoch erfolgten diesbezügliche Vermutungen durch die Ärzte der Irrenanstalt, auch wenn es zuvor nicht erwähnt worden war. ${ }^{73}$

Die Analyse der Ursachenfindung durch die Ärzte in der Anstalt zeigt jedoch nicht, wie andernorts erhoben, eine überdurchschnittlich häufige Annahme von Verlegenheitsursachen der Menstruation bei den Frauen $^{74}$ und Onanie bei den Männern. ${ }^{75}$ Auffälliger war hier der Unterschied in den Zuschreibungen des falsch entwickelten Geschlechts-

70 M13, Krankengeschichte 1835.

71 F1, Anamnese 1831.

72 Zur Geschichte des Diskurses über Onanie vgl. Franz X. Eder, Kultur der Begierde. Eine Geschichte der Sexualität (München 2002) 91-127.

73 M3, Anamnese 1831.

74 Vgl. Sylvelyn HÄHNER-ROMBACH, Arm, weiblich - wahnsinnig? Patientinnen der Königlichen Heilanstalt Zwiefalten im Spiegel der Einweisungsgutachten von 1812 bis 1871 (Zwiefalten 1995).

75 Vgl. Ann GOLDBERG, Psychiatrie und weibliche Devianz: Frauen, ländliche Gesellschaft und die nassauische Irrenanstalt Eberbach, 1815-1849. In: Christina VANJA, Steffen HAAS, Gabriela DEUTSCHLE, Wolfgang EIRUND, Peter SANDNER (Hg.), Wissen und Irren. Psychiatriegeschichte aus zwei Jahrhunderten - Eberbach und Eichberg (Kassel 1999) 76-93. Dies., Sex, Religion, and the Making of Modern Madness. The Eberbach Asylum and German Society, 1815-1849 (Oxford 2001). 
triebs. Vor allem aber lagen die geschlechtsspezifischen Unterschiede darin, dass die Ursachen bei den Frauen sehr häufig im Charakter und in den Eigenschaften gesehen wurden, während sich bei den Männern die verursachenden Eigenschaften, vorausgegangenen körperlichen Krankheiten und der kritisierte Lebenswandel die Waage hielten. Der Wert für den Lebenswandel als Auslöser bei den Frauen lag hingegen etwas unter dem der Männer.

\section{Fazit}

Die Untersuchung zeigte deutlich, dass wir es in Bezug auf psychische Krankheiten in der ersten Hälfte des 19. Jahrhunderts bei Laien und Ärzten mit unterschiedlichen Vorstellungsmodellen zu tun haben. Psychische Krankheit wurde von der Bevölkerung als von außen kommend wahrgenommen, während die Ärzte den Ursprung der Krankheit in den Menschen hinein verlegten. Sowohl Laien als auch Ärzte versuchten sich durch verschiedene Zuschreibungen von den Erkrankten abzugrenzen. Die Erklärungen für die Entstehung der Krankheiten korrespondierten mit den Wahrnehmungsmodellen: Bei den Laien überwog die Erklärung der Krankheit durch ein singuläres Ereignis, während die Ärzte die Ursachen im Menschen in Form von Dispositionen und Erziehungsfehlern suchten.

Besonders interessant ist der Vergleich mit der anfangs zitierten Studie Jodelets. Während sie Anzeichen dafür fand, dass psychische Krankheiten als ansteckend empfunden wurden, ließ sich dies in der vorliegenden Untersuchung überhaupt nicht erkennen. Dies könnte zum einen dadurch erklärt werden, dass die Krankheit durch die wahrgenommene Herkunft von außen sehr individuell an einem Menschen haftete, dieser speziellen Person zustieß. Da die Ursachen mit bestimmten Ereignissen verbunden waren, fürchtete man wohl eher „gefährliche“ Situationen und Umstände. Zum anderen waren die Zusammenhänge über Ansteckungen zwar schon durch die unterschiedlichen Epidemien wie auch im Hinblick auf Geschlechtskrankheiten etwas bekannt, jedoch dürften solche Vorstellungen im Alltagsleben noch nicht in dem Maße präsent gewesen sein, dass sie schon auf psychische Krankheiten umgelegt hätten werden können. Vor diesem Hintergrund wird die Flexibilität der Alltagsvorstellungen besonders plastisch: Die gegen Ende des 19. Jahrhunderts oder in der ersten Hälfte des 20. Jahrhunderts entwickelte Ansteckungsvorstellung von psychischen Krankheiten schuf die Möglichkeit, solchen Krankheiten auf neue Weise durch Vermeidung der „Ansteckungsgefahr" vorzubeugen. Dieser Prozess dürfte Hand in Hand mit der an der Oberfläche akzeptierten stärkeren Akzeptanz der wissenschaftlichen Denkweise vom Ursprung der Krankheit im Menschen erfolgt sein. Durch die Aufgabe der Vorstellung, dass psychische Krankheiten durch ganz konkrete Ereignisse hervorgerufen werden, musste ein neuer Schutz gesucht werden. 


\section{Abkürzungsverzeichnis}
A Anamnese
F Weiblich
KG Krankengeschichte
LD Laiendokumente
M Männlich
OZ Ordinationszettel (tägliche und monatliche Berichte)
PKH Psychiatrisches Krankenhaus Hall in Tirol

\section{Quellen}

Psychiatrisches Krankenhaus Hall (PKH)

Krankenakten 1830-1850.

Aufnahmebuch I: „Aufnahms Protocoll 1830-1863“.

\section{Literatur}

BREIT Stefan, „Leichtfertigkeit“ und ländliche Gesellschaft. Voreheliche Sexualität in der frühen Neuzeit (München 1991).

DIETRICH Elisabeth, Die Bevölkerungsentwicklung Tirols im ausgehenden 18. und im 19. Jahrhundert. In: Gesellschaft für Wirtschaftsdokumentationen Ges. m. b. H. (Hg.), Chronik der Tiroler Wirtschaft mit Sonderteil Südtirol (Wien 1992) I 125-139.

DIETRICH Elisabeth,MEIXNER, Wolfgang, Die wirtschaftspolitischen Rahmenbedingungen im ausgehenden 18. und beginnenden 19. Jahrhundert. In: Gesellschaft für Wirtschaftsdokumentationen Ges.m.b.H. (Hg.), Chronik der Tiroler Wirtschaft mit Sonderteil Südtirol (Wien 1992) I 115-124.

Eder Franz X., Kultur der Begierde. Eine Geschichte der Sexualität (München 2002) 91-127.

GOLDBERG Ann, Psychiatrie und weibliche Devianz: Frauen, ländliche Gesellschaft und die nassauische Irrenanstalt Eberbach, 1815-1849. In: Christina VANJA, Steffen Haas, Gabriela DEUTSCHLE, Wolfgang EIRUND \& Peter SANDNER (Hg.), Wissen und Irren. Psychiatriegeschichte aus zwei Jahrhunderten - Eberbach und Eichberg (Kassel 1999) 76-93.

GOLDBERG Ann, Sex, Religion, and the Making of Modern Madness. The Eberbach Asylum and German Society, 1815-1849 (Oxford 2001).

HÄHNER-ROMBACH Sylvelyn, Arm, weiblich - wahnsinnig? Patientinnen der Königlichen Heilanstalt Zwiefalten im Spiegel der Einweisungsgutachten von 1812 bis 1871 (Zwiefalten 1995).

HECHENBERGER Johann Georg, Grundriß zu einem Organon der spezifischen Seelenheilkunst, vorzüglich für die Landpraxis gemeinfaßlich dargestellt (Wien 1841).

HONEGGER, Claudia, Die Ordnung der Geschlechter. Die Wissenschaften vom Menschen und das Weib, 1750-1850 (Frankfurt a. M. 1991).

JÜTTE Robert, Geschichte der Alternativen Medizin. Von der Volksmedizin zu den unkonventionellen Therapien von heute (München 1996).

JODELET Denise, Madness and social representation (New York 1991).

MANTL Elisabeth, Heirat als Privileg. Obrigkeitliche Heiratsbeschränkungen in Tirol und Vorarlberg 1820 bis 1920 (Wien 1997).

MOSCOVICI Serge, Foreword. In: Claudine HERZLICH, Health and Illness: A Social Psychological Analysis (London 1973) XI-XIII.

DE ROSA Annamaria Silvana, The social representations of mental illness in children and adults. In: Willem DOISE, Serge MOSCOVICI (Hg.), Current Issues in European Social Psychology 2 (Cambridge 1987) 47-138.

SCHOTT Heinz, TÖLLE Rainer, Geschichte der Psychiatrie. Krankheitslehren, Irrwege, Behandlungsformen (München 2006) 20-22. 
STOLZ Josef, Bemerkungen über die Tirolische Landes-Irren-Anstalt zu Hall (Innsbruck 1869).

TSCHALLENER Johann, Beschreibung der k. k. Provinzial-Irren-Heilanstalt zu Hall in Tirol; mit Rücksicht auf die Statuten der Anstalt, auf die therapeutischen und psychologischen Grundsätze der Behandlung der Geisteskranken und auf ihre achtjährigen Resultate. Mit 19 Krankengeschichten und verschiedenen Andeutungen zum Wohl dieser Unglücklichen; nebst einem Anhange über die Anlage von Zimmern für Irre und Tobende (Innsbruck 1842).

WAGNER Wolfgang, DUVEEN Gerard, FARR Robert, JOVCHELOVITCH Sandra, LORENZI-CIOLDI Fabio, MARKOVÁ Ivana, ROSE Diana, Theory and method of social representations. In: Asian Journal of Social Psychology 2 (1999) 95-125.

WAGNER Wolfgang, HAYES Nicky, Everyday Discourse and Common Sense The Theory of Social Representations (Basingstoke, Hampshire 2005).

ZILLNER F. V., Ueber die größere Häufigkeit von Irrsinnsfällen unter der weiblichen Bevölkerung des Landes Salzburg. In: Mittheilungen der Salzburger Landeskunde (1879), 151-159. 
